\title{
Identifying genes that impact on aroma profiles produced by Saccharomyces cerevisiae and the production of higher alcohols
}

\author{
Gustav Styger • Dan Jacobson • Florian F. Bauer \\ Institute for Wine Biotechnology, Stellenbosch University, Stellenbosch \\ 7600 , South Africa. \\ The online version of this article contains supplementary material, \\ which is available to authorized users.
}

\begin{abstract}
During alcoholic fermentation, many volatile aroma compounds are formed by Saccharomyces cerevi siae, including esters, fatty acids, and higher alcohols. While the metabolic network that leads to the formation of these compounds is reasonably well mapped, surprisingly little is known about specific enzymes involved in specific reactions, the regulation of the network, and the physiological roles of individual pathways within the network. Furthermore, different yeast strains tend to produce significantly different aroma profiles. These differences are of tremendous biotechnological interest, since producers of alcoholic beverages such as wine and beer are searching for means to diversify and improve their product range. Various factors such as the redox, energy, and nutritional balance of a cell have previously been suggested to directly or indirectly affect and regulate the network. To gain a better understanding of the regulations and physiological role of this network, we screened a subset of the EUROSCARF strain deletion library for genes that, when deleted, would impact most significantly on the aroma profile produced under fermentative conditions. The 10 genes whose deletion impacted most significantly on higher alcohol production were selected and further characterized to assess their mode of action within or on this metabolic network. This is the first description of a large-scale screening approach using aroma production as the primary selection criteria, and the data suggest that many of the identified genes indeed play central and direct roles within the aroma production network of $S$. cerevisiae.
\end{abstract}

Keywords Ehrlich pathway · Branched chain amino acid metabolism $\cdot$ Higher alcohols $\cdot$ Wine aroma

\section{Introduction}

The production of aromatic or volatile compounds during alcoholic fermentation by yeast contributes significantly to the quality of products such as wine and beer. The most relevant families of compounds produced by yeast include esters, higher alcohols, and fatty acids. These compounds, and associated metabolites, play an important part in the flavor and aroma of wine (Mateo et al. 1998; Lambrechts and Pretorius 2000; Majdak et al. 2002), beer (Vanderhaegen et al. 2003; Trelea et al. 2004), cider (Vidrih and Hribar 1999), as well as that of fermented dairy products such as cheese (Thierry and Maillard 2002; Thierry et al. 2002). Different commercial wine and beer yeast strains produce significantly different aroma compounds, and such strains have in part been selected for their ability to impart specific sensory profiles to the final product (Romano et al. 2003; Estevez et al. 2004; Rossouw et al. 2008).

However, the metabolic network responsible for the production of these compounds (Fig. 1) is complex and, while reasonably well mapped, little information about the role of specific genes and their regulation within this network is available. Here we applied a mutant screening approach to identify genes whose products most significantly impact on this network. The primary focus of the work was directed towards identifying genetic factors that affect the conversion of amino acids to aroma compounds. Indeed, many datasets have confirmed that amino acids 


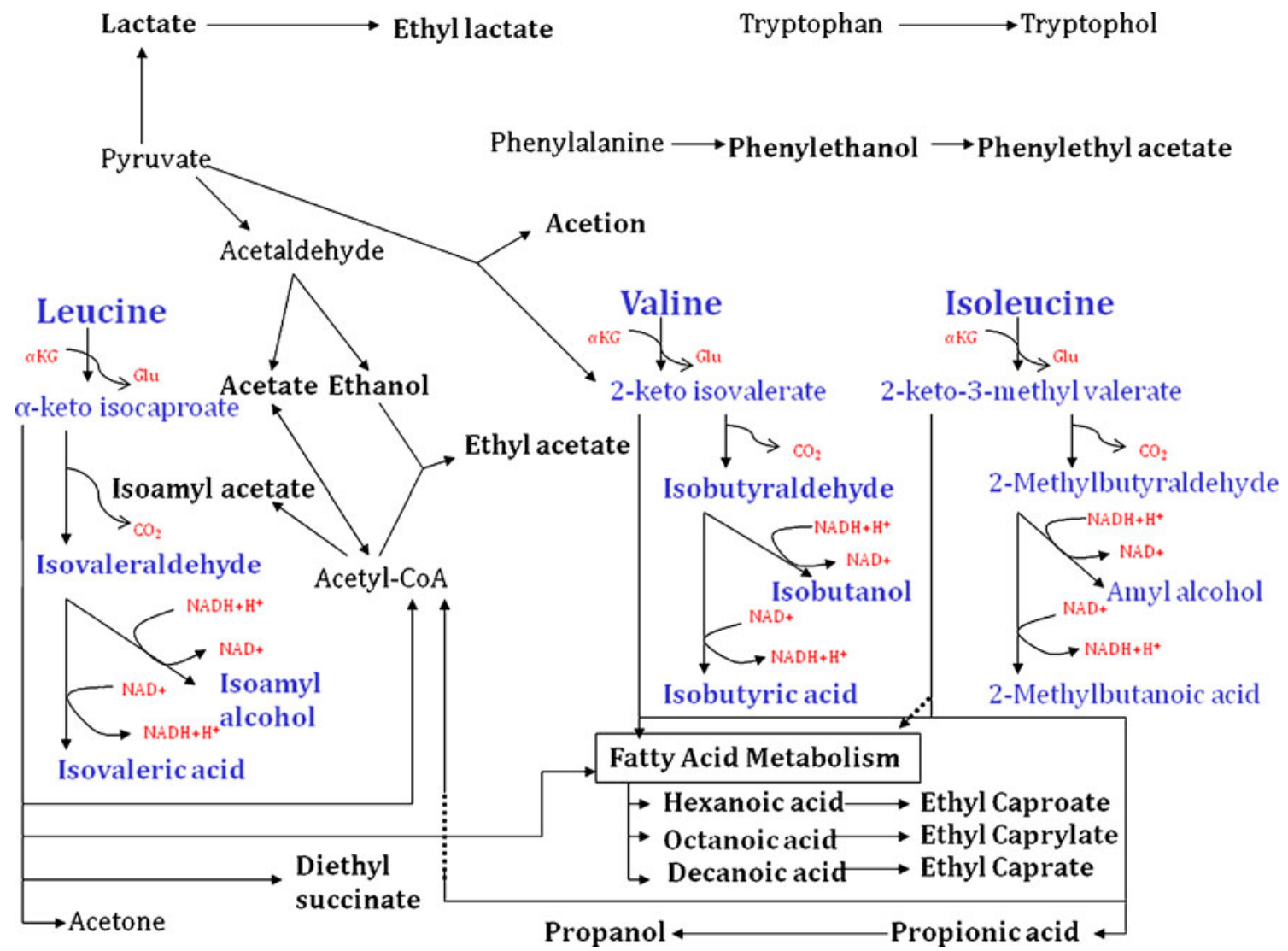

Fig. 1 A simplified metabolic map of yeast aroma compound production, indicating known metabolic linkages. Bold type indicates aroma compounds measured with GC FID during this study. Com pounds shown in blue color constitute a diagrammatical representation

of the Ehrlich pathway, responsible for the production of higher alcohols and volatile acids. Co factors and transition metabolites are shown in red. $\alpha K G \propto$ keto glutamate, Glu glutamate

constitute the most relevant precursors for the biosynthesis of many volatile compounds (Ardö 2006). The central part of this pathway is also referred to as the Ehrlich reaction (Hazelwood et al. 2008), and some aspects of the impact of this pathway on aroma production have been studied in the past (Dickinson et al. 1998, 2000, 2003; Eden et al. 2001).

During alcoholic fermentation, central carbon metabolism and associated pathways provide $\mathrm{C}_{2}, \mathrm{C}_{3}, \mathrm{C}_{4}$, and $\mathrm{C}_{5}$ carbon precursors such as pyruvate, oxalacetate, and $\alpha$ ketoglutarate. Amino acids, on the other hand, can be metabolized in several ways. In particular, they can be used as such for protein synthesis, or metabolized into other compounds and used for other purposes and metabolic processes, and serve in particular as providers of amino groups (Bauer and Pretorius 2000). When deaminated, the main product of the breakdown of these amino acids is a higher alcohol, or fusel alcohol, so called due to the fact that they have more carbon atoms than ethanol.

The branched chain amino acids, leucine, isoleucine, and valine for example, are transported into the yeast cell via several transport systems (Beltran et al. 2004; Grauslund et al. 1995). Under conditions of nitrogen limitation, these amino acids are used to support the synthesis of other nitrogen-containing metabolites. This transamination reaction is catalyzed by mitochondrial and cytosolic branched chain amino acid aminotransferases (BCAATases) encoded by the BAT1 and BAT2 genes (Eden et al. 1996, 2001; Kispal et al. 1996; Ben-Yosef et al. 1998; Prohl et al. 2000; Lilly et al. 2006). In this manner, $\alpha$-ketoisocaproic acid is formed from leucine, $\alpha$-ketoisovaleric acid from valine, and $\alpha$-keto- $\beta$-methylvaleric acid from isoleucine (Dickinson and Norte 1993; Dickinson et al. 1997, 1998, 2000). Yeast, however, can also generate these $\alpha$-keto acids through the so-called anabolic pathway, from glucose via pyruvate (Chen 1977; Dickinson and Norte 1993; Dickinson et al. 1997). However, Chen (1977) has shown that during a model beer fermentation, the Ehrlich pathway contributed $75 \%$ and $85 \%$, respectively, to the formation of their corresponding higher alcohols in the case of isoleucine and leucine.

The following step in the Ehrlich pathway is the decarboxylation of the $\alpha$-keto acid into an aldehyde (Jansen et al. 2003). Thus, isovaleraldehyde is formed from $\alpha$ ketoisocaproic acid, isobutyraldehyde from $\alpha$-ketoisovaleric 
acid, and 2-methyl butyraldehyde is formed from $\alpha$-keto- $\beta$ methylvaleric acid (Dickinson and Norte 1993; Dickinson et al. 1997, 2000; Dickinson 2000). It has been suggested that the pyruvate decarboxylase genes, $P D C 1, P D C 5$, and $P D C 6$, may play a part in this decarboxylation reaction, but they are not essential (Ter Schure et al. 1998; Yoshimoto et al. 2001). Other possible decarboxylase genes that could be involved in the decarboxylation of these $\alpha$-keto acids are KID1/THI3 and ARO10 (Dickinson et al. 2003; Vuralhan et al. 2003, 2005).

The Ehrlich pathway now splits in two and the final fate of the branched chain amino acid is thought to depend on the redox status of the yeast cell (Vuralhan et al. 2003). The aldehyde can either be reduced via a NADH-dependent reaction to its respective higher alcohol, i.e., isoamyl alcohol is formed from isovaleraldehyde, isobutanol is formed from isobutyraldehyde, and active amyl alcohol is formed from 2-methyl butyraldehyde (Dickinson and Norte 1993; Dickinson et al. 1997, 2000), or it can be oxidized via a $\mathrm{NAD}^{+}$-dependent reaction into a volatile acid. If this occurs, iso-valeric acid is formed from isovaleraldehyde, iso-butyric acid is formed from isobutyraldehyde, and 2methyl butanoic acid is formed from 2-methyl butyraldehyde (Dickinson and Norte 1993; Dickinson et al. 1997, 2000). It has been suggested that an alcohol dehydrogenase may catalyze this reductive reaction and an aldehyde dehydrogenase the oxidation reaction (Dickinson et al. 2003; Vuralhan et al. 2005).

The redox duality of these last two steps of the Ehrlich pathway has given rise to the hypothesis that higher alcohols or volatile acids are formed to help maintain the $\mathrm{NADH} / \mathrm{NAD}^{+}$ratio and so the redox balance of the cell (Van Dijken and Scheffers 1986; Quain 1988). Some authors, however, believe that the cell produces enough other redox equivalents such as ethanol, glycerol, acetate, acetaldehyde, and succinate to fulfill this role (Boulton et al. 1995). Another hypothesis for higher alcohol production is the removal of toxic aldehyde compounds or as an alternative source for the cell to obtain nitrogen (Boulton et al. 1995; Volbrecht and Radler 1973).

A previous work showed that the gene products of $B A T 1$ and $B A T 2$ were responsible for the first step in the pathway and that deletion of these genes had an impact on the formation of higher alcohols (Lilly et al. 2006). Changes in the concentrations of other, seemingly unrelated, aroma compounds were also observed, highlighting the complexities of the interconnections within such complex metabolic networks (Lilly et al. 2006). In order to identify genes that have the most important impact on the metabolic flux through this network, the yeast deletion library was used to screen for genes that might be involved in the different biochemical reactions of the pathway, i.e., decarboxylases, dehydrogenases, and reductases (Table 1). Genes whose deletion caused the strongest decrease in the production of
Table 1 Genes involved in the primary screen for possible involve ment in the Ehrlich pathway

\begin{tabular}{|c|c|c|c|c|c|}
\hline \multicolumn{3}{|c|}{ Dehydrogenases } & \multirow{2}{*}{$\begin{array}{l}\text { Decarboxylases } \\
\text { GAD1 }\end{array}$} & \multicolumn{2}{|c|}{ Reductases } \\
\hline$A A D 3$ & $G P D 1$ & MTD1 & & $A H P 1$ & MET16 \\
\hline$A A D 4$ & GPD2 & $O Y E 2$ & $G C V 1$ & $A Y R 1$ & $M X R 1$ \\
\hline$A A D 6$ & GUT2 & OYE3 & $G C V 2$ & $C B R 1$ & OARl \\
\hline$A D H 1$ & HIS4 & $P D A 1$ & $P A D 1$ & CORI & PRO2 \\
\hline$A D H 2$ & HOM2 & $P D B 1$ & $P D C 1$ & ECM17 & QCR2 \\
\hline$A D H 3$ & HOM6 & $P D X 1$ & $P D C 5$ & ERG24 & QCR 8 \\
\hline ADH4 & $I D H 1$ & PUT1 & PDC6 & $E R G 4$ & $R N R 1$ \\
\hline ADH5 & $\mathrm{IDH} 2$ & PUT2 & PSD2 & ETR1 & $R N R 3$ \\
\hline ADH6 & $I D P 1$ & $S D H 1$ & SPE1 & FRE1 & SPS19 \\
\hline$A D H 7$ & $I D P 2$ & $S D H 2$ & THI3 & FRE2 & $S R X 1$ \\
\hline$A L D 2$ & $I D P 3$ & $\mathrm{SDH} 4$ & & FRE3 & $T R R 2$ \\
\hline$A L D 3$ & $I M D 3$ & SER3 & & GRE3 & \\
\hline$A L D 4$ & IMD4 & SER33 & & $G R X 2$ & \\
\hline ALD5 & $K G D 1$ & SFA1 & & $H M G 1$ & \\
\hline ALD6 & $K G D 2$ & $T D H 1$ & & $H M G 2$ & \\
\hline$A R A 1$ & $L A T 1$ & $T D H 2$ & & $L Y S 2$ & \\
\hline$D L D 3$ & $L P D 1$ & $T D H 3$ & & LYS5 & \\
\hline$G D H 2$ & LYS12 & $T Y R 1$ & & $M C R 1$ & \\
\hline$G D H 3$ & $M D H 1$ & $U R A 1$ & & MET10 & \\
\hline$G N D 1$ & $\mathrm{MDH} 2$ & $X Y L 2$ & & MET12 & \\
\hline GND2 & MDH3 & $Z W F 1$ & & MET13 & \\
\hline
\end{tabular}

higher alcohols or related metabolites were further investigated. Thus, 10 genes were obtained whose products seem to be either directly or indirectly involved with the Ehrlich pathway. Further in-depth study of these genes was performed and the results show how they impact on the formation of higher alcohols and related compounds.

\section{Materials and methods}

Strains and growth conditions

All strains used where obtained from the EUROSCARF deletion library, except the $B A T 1$ and $B A T 2$ over-expression ( $p B A T 1$ and $p B A T 2$ ) and deletion strains (dbat1 and dbat2) that were previously generated (Lilly et al. 2006). The generation of deletions in the newly identified genes is described below. The media used throughout this study was synthetic complete dextrose (SCD) media (Sambrook et al. 1989), with the following changes or additions: SCD+++ medium contained $2 \%(w / v)$ glucose, standard concentrations of all amino acids, except increased concentrations $(150 \mathrm{mg} / \mathrm{l})$ of each of the branched chain amino acids, leucine, isoleucine, and valine. SCD5 medium contained standard concentrations of all amino acids, but 5\% $(w / v)$ glucose, whereas the SCD5+++ medium had the same 
increased concentrations of the branched chain amino acids as the $\mathrm{SCD}+++$ medium, but with a glucose content of $5 \%$ $(w / v)$. Initial screening experiments were done in duplicate, while follow-up experiments were performed in triplicate.

Strains were grown overnight in $5 \mathrm{ml}$ of YPD medium and inoculated into the sample culture at an optical density of 0.1 at $600 \mathrm{~nm}$. Sample cultures consisted of $80 \mathrm{ml}$ medium in $200 \mathrm{ml}$ glass bottles and were placed on a shaking incubator at $30^{\circ} \mathrm{C}$. The bottles were sealed with only tin foil and no fermentation caps, resulting in selfanaerobic growth. Weight loss was measured each day to determine the rate and stage of fermentation. After 7 days, a final OD was taken to ensure that the results could be normalized, and the cell suspension was centrifuged at $5,000 \mathrm{rpm}$ for $5 \mathrm{~min}$. The cell-free supernatant was used for gas chromatographic (GC) analysis.

Recombinant DNA and plasmid construction

Standard procedures for the isolation and manipulation of DNA were used throughout this study (Ausubel et al. 2003). Restriction enzymes, T4 DNA ligase (Roche), and ExTaq DNA polymerase (Fermentas) were used in the enzymatic manipulation of DNA according to the specifications of the supplier.

Deletion strains were generated by using the set of primers listed in Table 2. The yeast disruption plasmid,
YDp-U, containing the URA3 gene was used as template for PCR with primers designed to amplify the URA3 gene, with 50 base pair (bp) overhangs, specific to either the $5^{\prime}$ or 3' flanking sequences of the 10 genes, i.e., $A A D 6$, ADH3, GPD2, HOM2, OYE2, PAD1, PRO2, QCR2, SPE1, and THI3. The resulting disruption cassettes were transformed into BY4742 yeast cells, and successful transformants would insert a functional URA3 gene into the target gene. Transformants were selected on SC solid medium (2\% agar, Difco) without uracil. Correct integration of the cassette into the correct genomic region was confirmed with the use of PCR and primers complimentary to the flanking regions of the 10 genes of interest as well as nested primers binding inside the coding region of the URA3 gene (data not shown).

Gas chromatographic analysis

Extraction of volatile compounds from the cell-free supernatant was done by liquid-liquid extraction. To each sample of $5 \mathrm{ml}$ cell-free supernatant, $0.1 \mu \mathrm{l}$ of an internal standard (4-methyl-2-pentanol, $500 \mathrm{mg} / \mathrm{l}$ in $12 \%(\mathrm{v} / \mathrm{v})$ ethanol) was added. The mixture was extracted with $1 \mathrm{ml}$ of diethyl ether for $5 \mathrm{~min}$ in an ultrasonic bath, followed by centrifugation at 4,000 rpm for $3 \mathrm{~min}$. The diethyl ether layer was removed and dried on anhydrous sodium sulfate $\left(\mathrm{Na}_{2} \mathrm{SO}_{4}\right)$ before being injected into the gas chromatograph.

Table 2 Primers used in this study

\begin{tabular}{|c|c|}
\hline Primer name & Sequence \\
\hline YDp AAD6 F & 5' ATGGCTGATTTATTTGCTCCTGCTCCTGAACCATCTACAGGAATTCCCGGGGATCCGGTG 3' \\
\hline YDp AAD6 R & $5^{\prime}$ TTCAACAGGTTCCATTTACCTTGATAGATGCTAAAAGGGGGCTGCAGGTCGACGGATCCG 3' \\
\hline YDp ADH3 F & $5^{\prime}$ ATGTTGAGAACGTCAACATTGTTCACCAGGCGTGTCCAACGAATTCCCGGGGATCCGGTG 3' \\
\hline YDp ADH3 R & $5^{\prime}$ TATTATTTACTAGTATCGACGACGTATCTACCCAAAATCTGCTGCAGGTCGACGGATCCG 3' \\
\hline YDp GPD2 F & $5^{\prime}$ ATGCTTGCTGTCAGAAGATTAACAAGATACACATTCCTTAGAATTCCCGGGGATCCGGTG 3' \\
\hline YDp GPD2 R & 5' \\
\hline YDp HOM2 F & $5^{\prime}$ ATGGCTGGAAAGAAAATTGCTGGTGTTTTGGGTGCTACTGGAATTCCCGGGGATCCGGTG 3' \\
\hline YDp HOM2 R & 5' CCTTAAATCAAGTTTCTTGCTAGTAAGATTTCGGCAATCAGCTGCAGGTCGACGGATCCG 3' \\
\hline YDp OYE2 F & 5' ATGCCATTTGTTAAGGACTTTAAGCCACAAGCTTTGGGTGGAATTCCCGGGGATCCGGTG 3' \\
\hline YDp OYE2 R & 5' ATTTTTGTCCCAACCGAGTTTTGAGCTTCTTCGTACGTAGCTGCAGGTCGACGGATCCG 3' \\
\hline YDp PAD1 F & 5' ATGCTCCTATTTCCAAGAAGAACTAATATAGCCTTTTTCAGAATTCCCGGGGATCCGGTG 3' \\
\hline YDp PAD1 R & 5' GTTACTTGCTTTTTATTCCTTCCCAACGAGGAAAAGTGTCGCTGCAGGTCGACGGATCCG 3' \\
\hline YDp PRO2 F & 5' ATGTCCAGTTCACAACAAATAGCCAAAAATGCCCGTAAAGGAATTCCCGGGGATCCGGTG 3' \\
\hline YDp PRO2 R & 5' TATAATGTCACAGTCTTTATATCTAAATCCTTGTGAACAAGCTGCAGGTCGACGGATCCG 3' \\
\hline YDp QCR2 F & 5' ATGTTGTCAGCAGCTAGATTGCAATTTGCCCAGGGGTCAGGAATTCCCGGGGATCCGGTG 3' \\
\hline YDp QCR2 R & 5' CTCTTACAATTCGTCCAAATATGGCAAGTTGGAAACATCAGCTGCAGGTCGACGGATCCG 3' \\
\hline YDp SPE1 F & 5' \\
\hline YDp SPE1 R & 5' CAATCGAGTTCAGAGTCTATGTATACTATATCCGCAGTCTGCTGCAGGTCGACGGATCCG 3' \\
\hline YDp THI3 F & $5^{\prime}$ ATGAATTCTAGCTATACACAGAGATATGCACTGCCGAAGTGAATTCCCGGGGATCCGGTG 3' \\
\hline YDp THI3 R & 5' TCAGTATCCAACTTGATTTTTTTTTAGAAGTGGTTGGAATGCTGCAGGTCGACGGATCCG 3' \\
\hline
\end{tabular}

The region homologous to the corresponding genes are underlined 
The analysis of volatile compounds was carried out on a Hewlett Packard 6890 gas chromatograph equipped with a split-splitless injector and a flame ionization detector (FID). A DB-FFAP capillary GC column (Agilent Technologies, Little Falls, Wilmington, USA) with dimensions of $60 \mathrm{~m}$ length $\times 0.25 \mathrm{~mm}$ internal diameter with a $0.5-\mu \mathrm{m}$ film thickness, was used for separation. Hydrogen was used as the carrier gas at a constant flow rate of $3.3 \mathrm{ml} / \mathrm{min}$. The injector temperature was $200^{\circ} \mathrm{C}$, the split ratio $15: 1$, the split flow rate $49.5 \mathrm{ml} / \mathrm{min}$, and injection volume $3 \mu 1$. The FID was operated at $250^{\circ} \mathrm{C}$. The oven temperature program was as follows: $33^{\circ} \mathrm{C}(17 \mathrm{~min})$ to $240^{\circ} \mathrm{C}(5 \mathrm{~min})$ at $12^{\circ} \mathrm{C} /$ min. For each of the compounds analyzed, an internal calibration curve was constructed using known amounts of authentic standards. The internal standard and the chemicals were sourced from Merck (Cape Town, South Africa).

Investigation of metabolic interactions

In order to investigate the metabolic effects of gene deletions, a metabolic map of the formation of these compounds was created. The software program GenMapp (Dahlquist et al. 2002; Doniger et al. 2003) was used to modify and adapt the already existing map of isoleucine, leucine, and valine degradation from the Kyoto Encyclopaedia of Genes and Genomes. Although the main function of this program is to overlay microarray data onto metabolic pathways, it is also possible to design novel or specialist gene databases. The data obtained from the GC analysis of the different deletion strains were treated as follows: the ratio of deletion strain sample to the wild-type sample was calculated and transformed to $\log 2$ in order to represent up- and downward trends equally. Microarray visualization software from The Institute for Genomic Research, TMev, was used to visualize the GC data and to cluster deletion strains with regards to their metabolic profile. A gene database where the aroma compounds were treated as genes was compiled and linked to the metabolic map. Lastly, the transformed GC data were fitted onto the map and any possible causal interactions were investigated.

\section{Statistical analysis}

The statistical differences between the EUROSCARF deletion strains and the wild-type strain on the GC data were determined using Student's $t$ test and the $p$ value was calculated. A $p$ value of $\leq 0.05$ was used as the threshold for statistical significance. As the intent of this initial screen was to identify strains for further in-depth analysis, it was preferable to accept type I errors (false positives) rather than type II errors (false negatives). As such, no multiple testing correction was performed on this dataset. However, the statistical differences between the various selected deletion strains and the wild-type reference strain on the further in-depth analysis and GC data were determined using one-way ANOVA, followed by the Holm-Sidak post test to rigorously determine statistical variability between the different strains, and an adjusted $p$ value of $\leq 0.01$ was used as the threshold for statistical significance. Multivariate data analysis of the dataset in the form of principal component analysis (PCA) was performed with the Unscrambler software suite.

\section{Results}

Screening of selected deletion mutants

A previous work (Lilly et al. 2006) involving the deletion and over-expression of BAT genes showed immediate and clear effects on concentrations of higher alcohols and related compounds, as well as the overall aroma profiles, formed during small-scale batch fermentations in synthetic medium. In order to discover genes that had the same effect on the aroma profile of the fermented product and which might be involved in or impact on the Ehrlich pathway, it was decided to use the yeast deletion library to screen for mutants that have a significant effect on branched chain amino acid catabolism.

As discussed above, the Ehrlich reaction consists of various types of biochemical reactions, i.e., decarboxylase and oxidation/reduction reactions. It was therefore decided to implement a semi-targeted screening for genes that are involved in similar biochemical reactions and could thus be involved in the biochemical reactions making up this pathway. Table 1 lists the deletion strains that were selected for the primary screening procedure. The total number of deletion strains screened was 104, including 10 strains deleted for decarboxylase genes, 64 strains deleted for dehydrogenase genes, and 33 strains deleted for reductase genes. Several putative genes were also included in the screen, selected on the basis of sequence homology and the presence of common motifs, but none produced aroma profiles different from the wild-type strain (data not shown).

Apart from the expected effects on the production of higher alcohols and related compounds, the screening of deletion strains produced varied results regarding the production of other aroma and related compounds as shown in Fig. 2. Compounds indicated in boldface in Fig. 1 could be detected with our GC-FID analysis method, but it would seem that the minimal media in which fermentation was conducted were not conducive to the formation of a number of important aroma compounds, especially esters. Production of phenyl ethanol was widely affected and the strains produced a varied range of amounts compared to the wild 
Fig. 2 Scatter plot of the GC data from the entire deletion library screening dataset, indi cating the end levels of various chemical compounds directly, as well as indirectly related to branched chain amino acid me tabolism. The strains are not indicated on the graphs due to lack of space. The wild type levels are indicated by a red dot and the blue dots represent the 10 genes chosen for further study

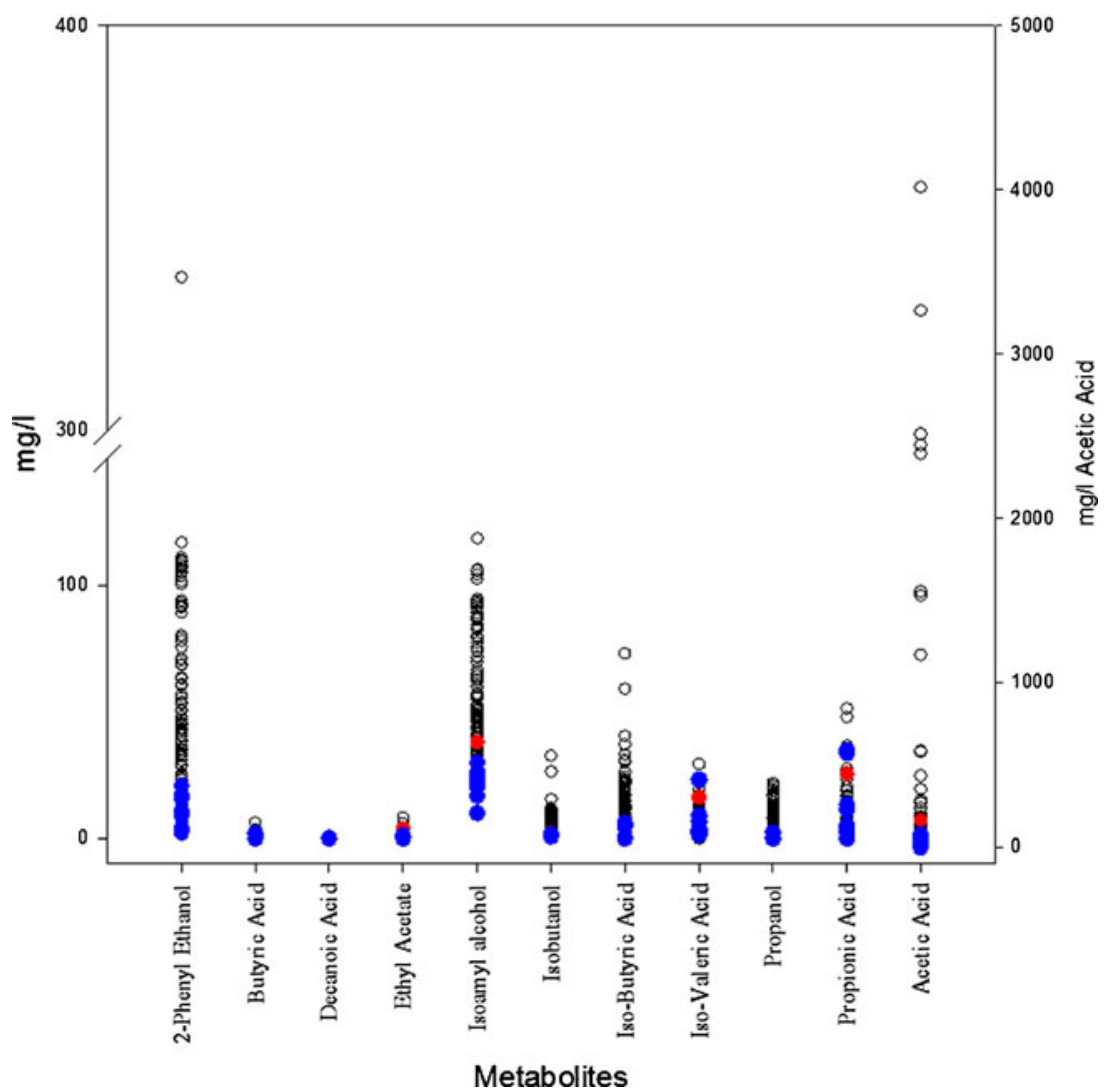

type. A range of production levels could also be seen for isoamyl alcohol and iso-butyric acid. The selected deletion strains are indicated in blue, with the wild-type strain in red, and it is clear that in most cases the selected strains produce less or similar amounts of a certain compound than the wild-type strain. A large range of acetic acid production is also observed, but was found to vary mostly in genes classified in the dehydrogenase functional category-likely due to redox effects resulting from gene deletions. Ethyl acetate is an important aroma ester and significantly lower levels were produced by most deletion strains across the functional categories, with the exception of one decarboxylase $(G C V 2)$ and one reductase (ECM17) strain. For the far downstream products of branched chain amino acid catabolism, propionic acid and propanol, a large spread of production levels was seen-from 0 in some instances to values in the range of $20-50 \mathrm{mg} / \mathrm{L}$. These results highlight the fact that selection for one or two criteria would not automatically result in a uniform distribution of attributes with regards to other compounds and again highlights the complexity of the metabolic network.

In order to focus on the analysis, the following criteria were used to select genes that appear to most strongly impact on the Ehrlich pathway: The deletion had to lead to large decreases in the production of higher alcohols such as isoamyl alcohol and isobutanol and be associated with decreases in either iso-valeric acid or iso-butyric acid. As higher alcohols are of particular importance in an oenological context, principal component analysis (Qlucore Omics Explorer v2.2) was performed on the amounts of the following compounds produced by each of the 104 deletion strains: 2-phenyl ethanol, isoamyl alcohol, isobutanol, propanol, and methanol. As can be seen from the loading plot (Fig. 3b), the higher alcohols cluster together (with the exception of methanol) and their largest effect is clearly along principal component 1 (PC1) which explains $58 \%$ of the variation on its own. We deemed it desirable to study a set of genes that produced the lowest amounts of higher alcohols and were as closely correlated to one another as possible. Thus, we selected the most extreme gene deletions in this regard as represented by those present at the left hand tip of PC1 (colored red in the score plot, Fig. 3a). In addition, we selected pBAT1 and pBAT2, transaminase over-expression strains, as well as dbat2 the BAT2 deletion strain (also colored red in the score plot) even though it was not in this PC1 tip group to play the role of both positive and negative controls.

The genes that best matched these criteria were further investigated. The GC data for the major volatile metabolites produced by these selected deletion strains in the standard growth conditions are represented as a heat map in Fig. 4. The results represent the average of duplicate biological 


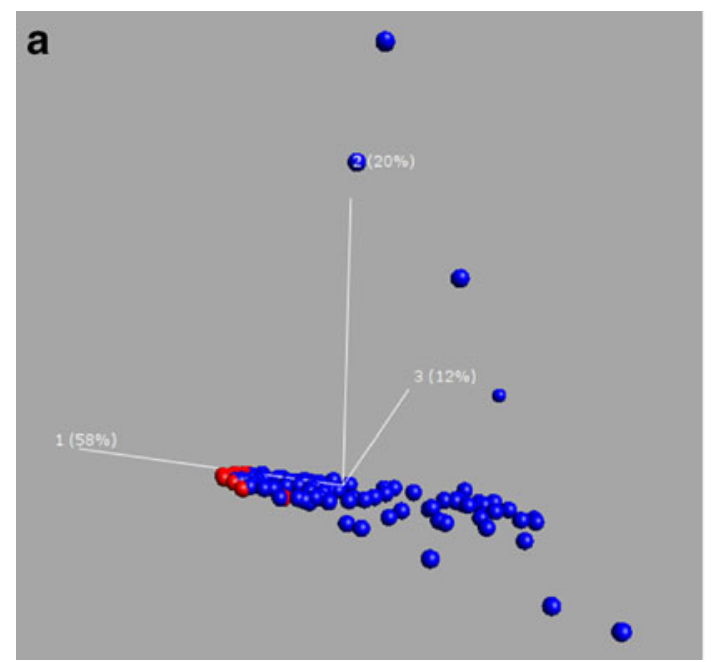

Fig. 3 Principal component analysis on the amounts of the following compounds produced by each of the 104 deletion strains: 2 phenyl ethanol, isoamyl alcohol, isobutanol, propanol, and methanol. The loading plot is indicated in $\mathbf{b}$, showing that the higher alcohols cluster together and their largest affect is clearly along principal component 1

repeats. Table 3 contains the results of the $t$ tests performed with pairwise comparisons between the wild-type and selected deletion strains. All strains producing a $p$ value of less than 0.05 were considered to be statistically significantly different and those not meeting this criterion are indicated in bold in Table 3. The statistical analysis of the complete screening dataset can be seen in the Supplemental material. As described above in the "Statistical analysis" of the "Materials and methods" section, no multiple testing correction was performed on this dataset.

The suggested metabolic functions and locations of the 10 candidate genes are shown in Table 4. Of the 10 genes selected, 5 are classified as dehydrogenases, 3 as decarboxylases, and 2 as reductases. The dehydrogenase genes include genes involved in major cellular redox maintenance reactions, such as GPD2 and $A D H 3$, as well as general redox reactions in the case of OYE2. This is an indication of the importance of redox homeostasis on the formation of these compounds. The remaining dehydrogenases, AAD6 and $H O M 2$, do not play a significant role in this regard and are possibly directly involved in the Ehrlich reaction. The three decarboxylases, PADI, SPE1, and THI3, catalyze reactions with substrates similar to those of the Ehrlich pathway and might exert their function directly on the pathway, or as promiscuous enzymes. The same could also apply for the two reductase genes, PRO2 and QCR2.

Figure 4 clearly indicates that all 10 genes have a significant impact on the majority of metabolites measured. In most strains, acetic acid and ethyl acetate production was significantly reduced, suggesting that the gene products

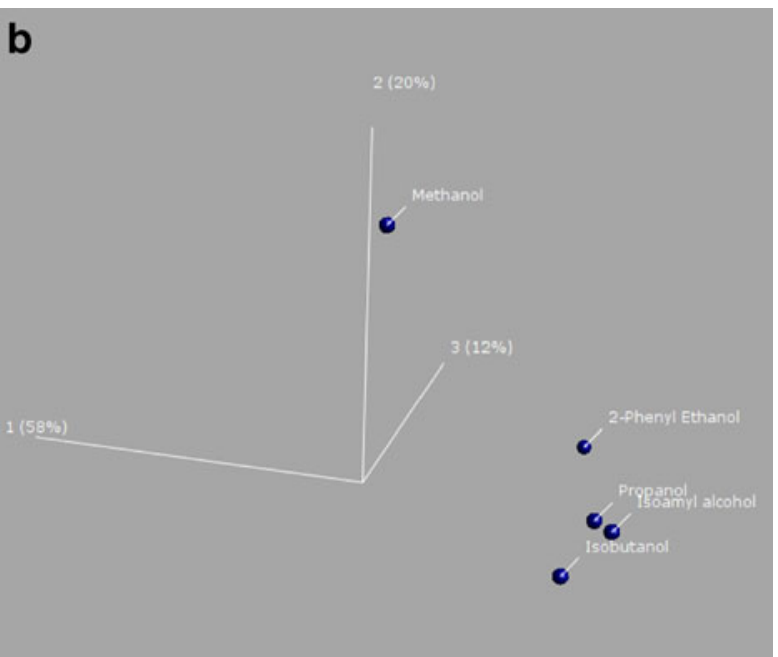

(PC1) which explains $58 \%$ of the variation on its own. The selected genes are represented by red dots and represent the genes whose deletion had the strongest negative impact on the concentrations of the higher alcohols in a

may impact on the redox balancing potential of the corresponding strains. All selected strains also showed a decrease in the level of isoamyl alcohol and isobutanol produced when compared to the wild-type strain, with the HOM2 deleted strain showing a fourfold decrease. Indeed, the levels of most of the deletion strains chosen are near to that of the BAT2 deletion strain, dbat2, that was previously shown to have a significant effect on the production of higher alcohols and their related metabolites (Lilly et al. 2006). In contrast, the $B A T 2$ over-expression strain, $p B A T 2$, shows an increase in the concentrations of these compounds, with little effect seen for the $B A T 1$ over-expression strain, $p B A T 1$, as well as the $B A T 1$ deletion strain, dbat1. This is in line with previous data (Lilly et al. 2006).

The effects on the production of the volatile branched chain acids are less clear-cut, especially in the case of isobutyric acid, where only three deletion strains, $\Delta$ hom 2 , $\Delta q c r 2$, and $\Delta$ thi3 show pronounced effects. However, all deletion strains except $\Delta q c r 2$ show a decrease in the amount of iso-valeric acid production, hinting at a possible specificity of certain gene products to certain metabolites in the Ehrlich pathway.

Since the initial screening experiment described above was performed using deletion strains from the EUROSCARF library, it was important to independently verify the results by deleting the 10 selected genes and re-assess their impact on the metabolism of higher alcohols and related compounds. Indeed, several reports and findings in our own laboratory suggest that a significant number of phenotypes of EUROSCARF deletion strains may not be directly due to 
Fig. 4 Heat map of the GC data of the 10 candidate strains, as well as certain control strains, indicating the levels of metabo lites with regards to the wild type strain as the $\log 2$ of their ratio. An increase in the pro duction of a certain metabolite is indicated by a red square and the intensity of the color repre sents the $\log 2$ fold value as indicated by the scale on the right hand side. A decrease is represented by a green square

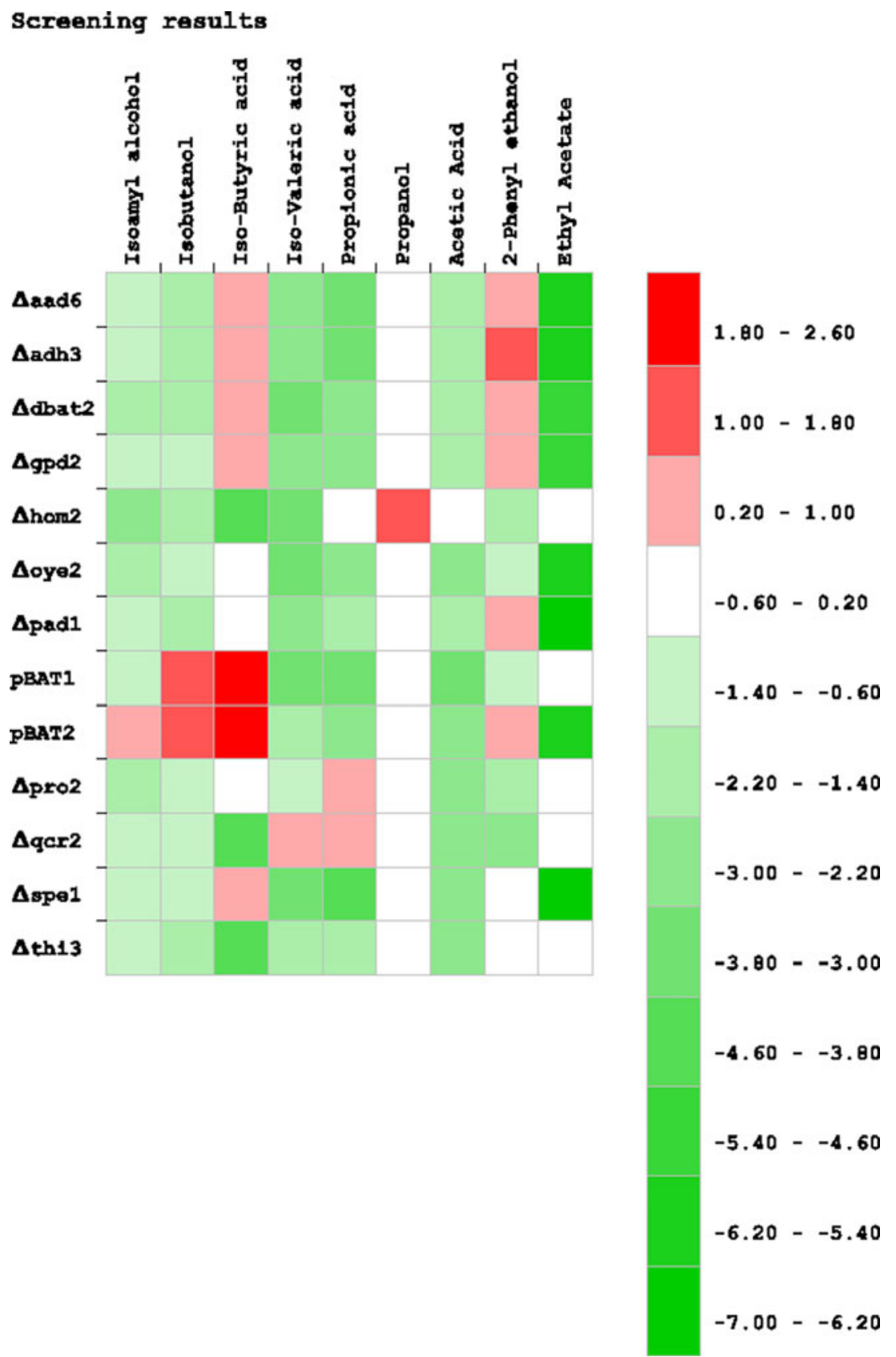

the deleted gene, but may be due to secondary mutations in those strains.

The resulting comparisons between the EUROSCARF strains and independently deleted strains can be seen in Fig. 5. The EUROSCARF strains are denoted by capitalized letters, while the independent strains are notated in small case with a preceding $\Delta$ sign. The radar plots in Fig. 5 indicate the ratio in $\log 2$ scale of the contribution of each compound to the total aroma profile of the strain compared to the respective wild-type control strain, and as such, it is easy to compare the distribution of certain aroma compounds across different strains.
In all cases, except in the case of $\Delta q c r 2$ (where an increase in the levels of Ehrlich metabolites is observed), the results confirmed those obtained with the EUROSCARF strains, both from a quantitative and qualitative perspective. This suggests that the $Q C R 2$ deletion strain in the EUROSCARF library either carries some secondary mutations that affect aroma production, or that the deletion of QCR2 has some impact on neighboring genes. As seen with the EUROSCARF deletion library, deletion of HOM2 had the most important effect on the final levels of both isoamyl alcohol and isobutanol, as well as on the concentrations of iso-butyric and iso-valeric acid. However, it is 
clear that there are some discrepancies between the two sets of strains where non-Ehrlich pathway metabolites are concerned, i.e., hexanoic acid and hexyl acetate. However, these compounds are produced in minute quantities in these strains, and as such, these small differences can be amplified by the use of $\log 2$ scaling. For all biological intents and purposes, these strains can be perceived to be very closely related in terms of the production of Ehrlich reaction and related metabolites.

\section{Aroma production in high sugar environments}

Most industrial fermentations take place in high sugar environments. For logistical reasons, our initial screen was carried out at laboratory conditions. In order to further verify that the candidate genes indeed influence the metabolites and end products of the Ehrlich pathway in fermentative conditions, the deletion strains were reassessed in smallscale batch fermentations with a glucose concentration of $5 \%$ $(w / v)$, and strains were grown in medium with (SCD5+++) and without (SCD5) added amounts of the three branched chain amino acids: leucine, isoleucine, and valine. All fermentations were carried out in triplicate.

These results confirm the data from the large-scale screening, i.e., that the deletion of the genes in each case led to a decrease in the amounts of higher alcohols and related compounds formed during the fermentation (Tables 5 and 6). The data also show that all strains continue to display fermentation kinetics similar to the WT, suggesting that the observed effects were not due to some indirect impact on growth (Supplemental material).

In Fig. 6, the final concentrations of the end products of the Ehrlich pathway for the deletion strains grown in SCD5 and SCD5+++ medium can be seen. Once again, it is clear from Fig. 6 (top panel) that the over-expression of the BAT2 gene leads to a 1.3-fold increase in the level of isobutanol produced. It is interesting to note that in this instance the effect on the concentration of isoamyl alcohol is much less pronounced than in the initial screen where the total glucose concentration in the medium was $2 \mathrm{~g} / \mathrm{l}(\mathrm{w} / \mathrm{v})$, compared to the SCD5 medium with a higher glucose concentration of $5 \mathrm{~g} / \mathrm{l}(w / v)$. It is possible that the higher glucose concentration may suppress the catabolism of specific branched chain amino acids to some extent.

All the deletion strains, except $\Delta a d h 3$, show a decrease in the levels of both isoamyl alcohol and isobutanol when compared to the wild-type yeast strain when grown in SCD5 medium. These decreases are in the same range, or lower than the decreases seen with the dbat 2 strain. This strongly suggests that these genes, with the possible exception of $\Delta a d h 3$, impacts on the conversion of the branched chain amino acids into higher alcohols in fermentative conditions. 
Table 4 List of the 10 candidate genes chosen after the initial screen and their attributes

\begin{tabular}{|c|c|c|c|c|}
\hline Gene & ORF & Function & Biological process & Cellular component \\
\hline$A A D 6$ & YFL056C & Aryl alcohol dehydrogenase (putative) & Aldehyde metabolism & Unknown \\
\hline$A D H 3$ & YMR083W & Alcohol dehydrogenase isoenzyme III & NADH oxidation, fermentation & Mitochondrion \\
\hline GPD2 & YOL059W & Glycerol 3 phosphate dehydrogenase & NADH oxidation, glycerol metabolism & Cytosol, mitochondrion \\
\hline HOM2 & YDR158W & Aspartic $\beta$ semi aldehyde dehydrogenase & $\begin{array}{l}\text { Homoserine biosynthesis, methionine } \\
\text { and threonine metabolism }\end{array}$ & Cytoplasm, nucleus \\
\hline OYE2 & YHR179W & $\begin{array}{l}\text { NAPDH dehydrogenase (old yellow } \\
\text { enzyme), isoform II }\end{array}$ & Unknown & $\begin{array}{l}\text { Cytoplasm, nucleus, } \\
\text { mitochondrion }\end{array}$ \\
\hline PAD1 & YDR538W & Phenylacrylic acid decarboxylase & Aromatic compound metabolism & Mitochondrion \\
\hline $\mathrm{PRO} 2$ & YOR323C & $\Gamma$ Glutamyl phosphate reductase & Proline biosynthesis & Cytoplasm, nucleus \\
\hline$Q C R 2$ & YPR191W & $\begin{array}{l}40 \mathrm{kDa} \text { ubiquinol cytochrome } c \text { reductase } \\
\text { core protein II }\end{array}$ & $\begin{array}{l}\text { Aerobic respiration, mitochondrial electron } \\
\text { transport }\end{array}$ & Mitochondrion \\
\hline SPE1 & YKL184W & Ornithine decarboxylase & Pantothenate, putrescine biosynthesis & Cytoplasm \\
\hline THI3 & YDL080C & $\alpha$ Ketoisocaproate decarboxylase & Thiamin biosynthesis & Nucleus \\
\hline
\end{tabular}

As expected, the level of isobutanol produced by the overexpression strain is increased tremendously when grown in branched chain amino acid-enriched medium, SCD5+++, although once again this effect was not seen with isoamyl alcohol. However, all the deletion strains showed decreases in the final concentrations of these two higher alcohols, with the $\Delta$ hom 2 deletion giving the biggest effect.

The scenario regarding volatile fatty acids is less clear. In the SCD5 medium (Fig. 6, bottom panel), the BAT2 overexpression strain and the strains $\Delta a d h 3, \Delta g p d 2, \Delta o y e 2$, $\Delta$ spel, and $\Delta$ thi3 all show an increase in the levels of isobutyric acid. The concentrations of iso-butyric acid are reduced in the remainder of the deletion strains. In the case of iso-valeric acid, however, all the deletion strains except for $\Delta$ thi3 show a marked drop in the levels produced.

Of the strains grown in the SCD5+++ medium (Fig. 6, bottom panel), the BAT1 and BAT2 over-expression strains,
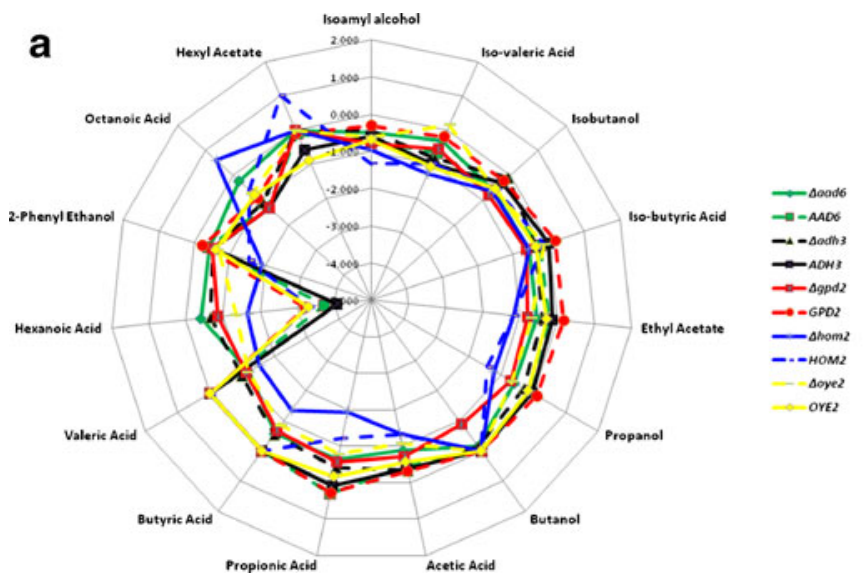

Fig. 5 Radar plots of the effect on the aroma profile produced by selected strains from the EUROSCARF deletion library vs. the strains where the same genes have been independently disrupted. EURO SCARF strains are denoted by capitalized letters, while the indepen dent strains are notated in italicized small case. a Deletion strains as well as $\Delta g p d 2, \Delta p r o 2$, and $\Delta q c r 2$, have elevated isobutyric acid levels when compared to that of wild-type strain. All the other deletion strains show a decrease in the concentration of this compound. The effect on the production of iso-valeric acid is much simpler, with all the deletion strains, except $\Delta$ pro 2 giving less or similar levels than for the wild-type strain.

The effect of gene deletion on the production of other aroma-related metabolites

From previous work where the BAT genes were perturbed (Lilly et al. 2006), it was observed that not only were concentrations of metabolites directly linked to these genes affected, but also other aroma metabolites not directly related to the higher alcohols. The metabolic map shown in Fig. 1 was used to generate schematic diagrams of the

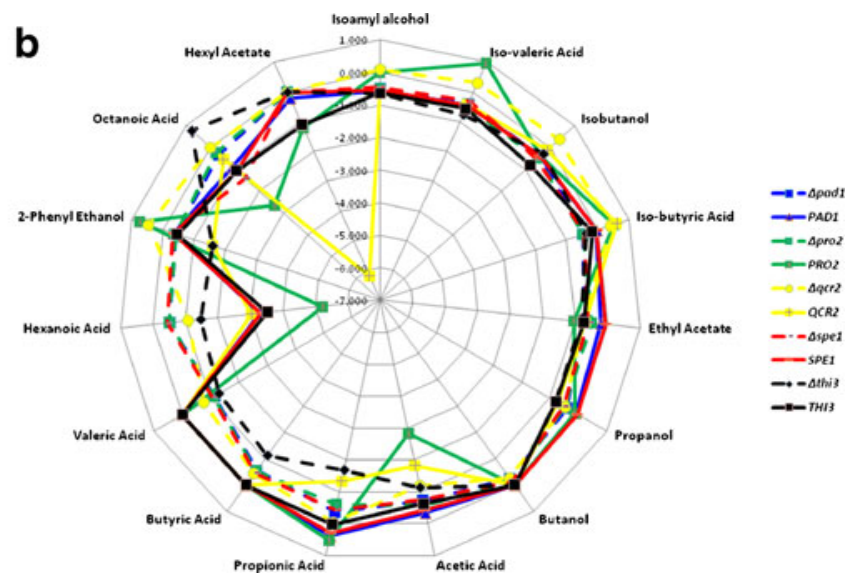

involving the following genes AAD6, ADH3, GPD2, HOM2, and $O Y E 2$; b data from the following deletion strains: PAD1, PRO2, QCR2, SPE1, and THI3. Values plotted are the $\log 2$ ratios of deletion strain vs. WT (BY4742) 
Table 5 Gas chromatographic data for deletion strains and control over expression strain grown for 1 week in SCD5 medium

\begin{tabular}{|c|c|c|c|c|c|c|c|c|c|c|}
\hline \multirow{2}{*}{$\begin{array}{l}\text { SCD5 } \\
\text { ID }\end{array}$} & \multicolumn{10}{|l|}{$\mathrm{mg} / 1$} \\
\hline & $\begin{array}{l}2 \text { Phenyl } \\
\text { ethanol }\end{array}$ & Acetic acid & Butanol & $\begin{array}{l}\text { Ethyl } \\
\text { acetate }\end{array}$ & $\begin{array}{l}\text { Hexyl } \\
\text { acetate }\end{array}$ & $\begin{array}{l}\text { Isoamyl } \\
\text { alcohol }\end{array}$ & Isobutanol & $\begin{array}{l}\text { Iso butyric } \\
\text { acid }\end{array}$ & $\begin{array}{l}\text { Iso valeric } \\
\text { acid }\end{array}$ & $\begin{array}{l}\text { Propionic } \\
\text { acid }\end{array}$ \\
\hline$A A D 6$ & $16.75 \pm 3.04$ & $116.92 \pm 66.24$ & $0.99 \pm 0.27$ & $5.37 \pm 1.08$ & $1.84 \pm 0.28$ & $49.67 \pm 6.48$ & $52.13 \pm 2.33$ & $1.26 \pm 0.41$ & $0.52 \pm 0.07$ & $3.94 \pm 1.38$ \\
\hline$A D H 3$ & $16.27 \pm 5.66$ & $66.07 \pm 15.12$ & $0.91 \pm 0.02$ & $4.78 \pm 0.54$ & $1.61 \pm 1.28$ & $57.17 \pm 11.57$ & $108.49 \pm 40.07$ & $1.77 \pm 0.61$ & $0.64 \pm 0.29$ & $4.1 \pm 1.14$ \\
\hline dbat1 & $10.24 \pm 0.01$ & $28.89 \pm 0.14$ & $0.92 \pm 0$ & $4.34 \pm 0.09$ & $1.4 \pm 0.01$ & $39.11 \pm 0.78$ & $27.82 \pm 0.38$ & $0.57 \pm 0$ & $0.24 \pm 0$ & $2.07 \pm 0.03$ \\
\hline dbat2 & $11.37 \pm 1.79$ & $64.7 \pm 36.29$ & $0.89 \pm 0.13$ & $3.7 \pm 1.29$ & $1.19 \pm 0.61$ & $39.36 \pm 4.49$ & $48.36 \pm 10.68$ & $0.7 \pm 0.13$ & $0.37 \pm 0.17$ & $2.36 \pm 0.55$ \\
\hline$G P D 2$ & $17.55 \pm 7.22$ & $1,936.04 \pm 18.97$ & $1.44 \pm 0.31$ & $7.47 \pm 5.58$ & $9.7 \pm 8.7$ & $41.51 \pm 2.59$ & $50.81 \pm 13.72$ & $2.76 \pm 0.44$ & $0.5 \pm 0.16$ & $3.15 \pm 0.46$ \\
\hline HOM2 & $8.7 \pm 0.5$ & $90.29 \pm 92.88$ & $0.81 \pm 0.22$ & $3.68 \pm 0.71$ & $1.39 \pm 0.2$ & $31.19 \pm 1.37$ & $36.5 \pm 4.28$ & $0.69 \pm 0.21$ & $0.31 \pm 0.1$ & $2 \pm 0.66$ \\
\hline OYE2 & $13.68 \pm 3.76$ & $1,883.75 \pm 4.53$ & $1 \pm 0$ & $6.87 \pm 4.47$ & $8.69 \pm 5.04$ & $39.03 \pm 8.99$ & $75.3 \pm 14.93$ & $2.32 \pm 0.22$ & $0.47 \pm 0.25$ & $5.62 \pm 1.92$ \\
\hline PAD1 & $9.38 \pm 0.83$ & $1,047.83 \pm 949.38$ & $0.95 \pm 0.25$ & $4.32 \pm 1.63$ & $2.56 \pm 1.72$ & $34.59 \pm 18.89$ & $42.92 \pm 8.81$ & $0.96 \pm 0.3$ & $0.38 \pm 0.06$ & $2.56 \pm 0.82$ \\
\hline$p B A T 1$ & $12.28 \pm 3.21$ & $237.09 \pm 186.86$ & $1.05 \pm 0.21$ & $5.66 \pm 0.71$ & $3.01 \pm 1.95$ & $55.08 \pm 4.24$ & $72.88 \pm 10.56$ & $1.14 \pm 0.09$ & $0.42 \pm 0.09$ & $4.1 \pm 0.63$ \\
\hline$p B A T 2$ & $15.34 \pm 1.69$ & $61.82 \pm 44.2$ & $1.45 \pm 0.67$ & $4.69 \pm 1.63$ & $1.48 \pm 1.09$ & $79.29 \pm 5.87$ & $159.53 \pm 54.6$ & $2.53 \pm 1.04$ & $0.57 \pm 0.09$ & $3.07 \pm 1.62$ \\
\hline PRO2 & $12.29 \pm 3.53$ & $120.96 \pm 65.61$ & $0.94 \pm 0.15$ & $4.28 \pm 1.72$ & $1.68 \pm 0.85$ & $44.72 \pm 5.72$ & $50.22 \pm 16.99$ & $0.93 \pm 0.38$ & $0.43 \pm 0.21$ & $2.97 \pm 0.39$ \\
\hline QCR2 & $10.46 \pm 2.04$ & $85.52 \pm 58.74$ & $0.85 \pm 0.15$ & $4.1 \pm 0.72$ & $0.89 \pm 0.78$ & $44.18 \pm 6.5$ & $41.36 \pm 5.6$ & $0.88 \pm 0.39$ & $0.44 \pm 0.26$ & $2.36 \pm 1.19$ \\
\hline SPE1 & $12.47 \pm 4.01$ & $662.25 \pm 832.64$ & $0.93 \pm 0.61$ & $6.23 \pm 4.35$ & $6.79 \pm 8.28$ & $37.78 \pm 2.58$ & $45.6 \pm 10.75$ & $1.54 \pm 1.18$ & $0.42 \pm 0.15$ & $2.64 \pm 0.76$ \\
\hline THI3 & $20.4 \pm 5.48$ & $545.96 \pm 941.9$ & $0.62 \pm 0.56$ & $3.78 \pm 5.57$ & $0.59 \pm 0.76$ & $47.29 \pm 7.75$ & $47.57 \pm 13.09$ & $2.91 \pm 1.58$ & $0.99 \pm 0.26$ & $5.31 \pm 3.6$ \\
\hline$W T$ & $17.12 \pm 3.78$ & $1,378.71 \pm 1,450.33$ & $1.29 \pm 0.33$ & $7.96 \pm 1.37$ & $4.05 \pm 3.44$ & $69.71 \pm 9.3$ & $116.81 \pm 35.74$ & $1.36 \pm 0.59$ & $1.08 \pm 0.6$ & $8.1 \pm 5.58$ \\
\hline
\end{tabular}

The results are the average of three replicates \pm the standard deviation

changes in aroma profile due to gene deletion. The ratio of all measured aroma compound from wild-type compared to the deletion strain was calculated and transformed by $\log 2$ to ensure an even spread of the fold differences. These values were then represented in the thickness of the arrow as seen in Fig. 7. Only the most relevant strains are shown in Fig. 7.

The effect of the $\Delta g p d 2$ strain on the aroma profile is seen in Fig. 7a. As Gpd2p is unlikely to play a direct role in the Ehrlich pathway, the effects caused by the deletion of the GPD2 gene on the aroma profile is likely due to be indirect and linked to the maintenance of redox homeostasis. That the aroma profile is in any case affected by the deletion of the GPD2 gene shows that the yeast aroma production pathway and especially the Ehrlich pathway are indeed sensitive to redox homeostasis.

The other three genes that impacted the most on the end products of the Ehrlich pathway-AAD6 (Fig. 7b), HOM2 (Fig. 7c), and THI3 (Fig. 7d) - are unlikely to have any impact on the global redox balance of the cell and therefore their impact on the Ehrlich pathway is much more likely to be a direct one. It is also interesting to note that deletion of all four genes had a huge negative impact on the concentration of decanoic acid and to a lesser extent that of octanoic acid.

Acetate production plays an important role in cellular redox maintenance. The effect of gene deletion on the production of this compound is very much dependent on medium composition with decreases in concentration seen in strains grown in the SCD5 medium (Table 5). In line with the role of acetate production, the levels of this compound are affected by the deletion of reductases and dehydrogenases, i.e., genes whose gene products are involved in reactions affecting the $\mathrm{NAD}^{+} / \mathrm{NADH}$ ratio. This once again reflects the sensitivity of the aroma production pathway towards perturbations in cellular redox homeostasis.

Figure 7 shows that only two esters are affected by the deletions of the selected genes, i.e., ethyl acetate and hexyl acetate. These acetate esters are synthesized by acetyltransferase enzymes which react with acetyl coenzyme A (acetylCoA), derived from either pyruvate, acetaldehyde, or acetate and, depending on the degree of affinity, with various higher alcohols to produce esters (Lilly et al. 2000). In most cases, the concentrations of these esters are negatively influenced by gene deletion, the only significant exception being the slight increase of ethyl acetate seen in the $\Delta g p d 2$ strain. The decrease in the concentration of these two acetate esters can be directly linked to the phenomenon of decreased acetate production. Less acetate would lead to less acetyl-CoA being present and thus to less esters being formed.

Another compound that is affected by the deletion of the selected genes (as seen in Fig. 7) and that also plays an important part in the aroma profile is 2-phenyl ethanol. This higher alcohol is formed from the aromatic amino acid phenylalanine, also via the Ehrlich pathway. Previous work (Van Dijken and Scheffers 1986; Vuralhan et al. 2003, 2005) showed that various genes can catalyze the conversion of aromatic amino acids into higher alcohols, including some involved in this study. It is thus likely that a deletion that has 


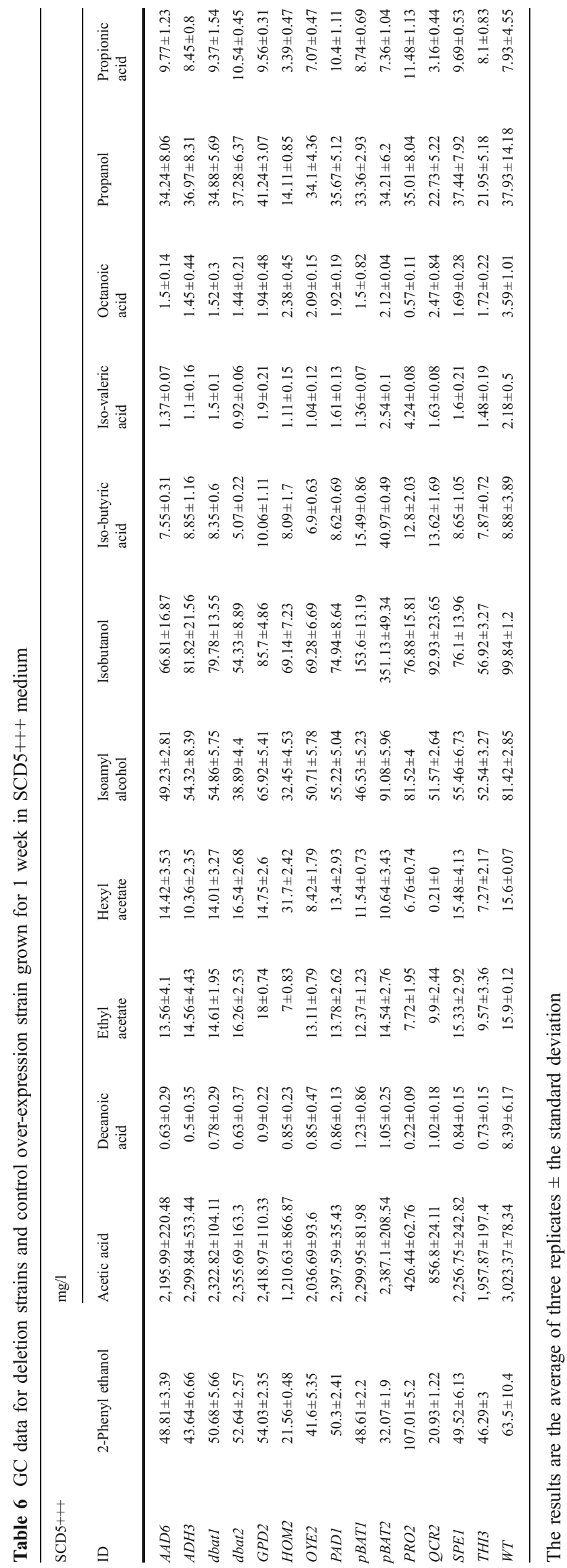

an effect of decreasing the concentrations of branched chain amino acid-derived higher alcohols would also decrease the concentrations of other compounds that are formed similarly.

Multivariate data analysis

Multivariate analysis of the dataset in the form of PCA was performed in order to investigate the factors contributing to the variance between the candidate genes and the control strains. The first and second components, $\mathrm{PC} 1$ and $\mathrm{PC} 2$, were able to explain $32 \%$ and $23 \%$, respectively, of the observed variance between the deletion strains. Figure $8 \mathrm{a}$ illustrates the bi-plot of $\mathrm{PC} 1$ vs. $\mathrm{PC} 2$ and shows that the deletion strains are spread out along the first component axis, with the two over-expression strains, $p B A T 1$ and $p B A T 2$, clustering together towards the bottom right-hand corner of the bi-plot, with the compounds most involved in explaining this distance to the other strains being isobutanol, iso-butyric acid, isoamyl alcohol, and octanoic acid (Fig. 8d).

A closer look along the PC1 axis shows that the three deletion strains $\Delta a a d 6, \Delta a d h 3$, and $\Delta g p d 2$ formed a distinct cluster. The compounds most responsible for this cluster are 2-phenyl ethanol, butyric acid, hexanoic acid, and acetate (Fig. 8b). ADH3 and GPD2 are major regulators of redox balancing under fermentative conditions, the fact that $\Delta a a d 6$ clusters together with such genes suggests that this gene plays a direct role in the Ehrlich pathway.

Figure $8 \mathrm{c}$ also shows that compounds such as propanol, valeric acid, diethyl succinate, iso-valeric acid, and propionic acid are important in explaining the difference between $\Delta$ thi3 and $\Delta h o m 2$ and the other selected mutants. It is interesting that these two genes cluster close to one another as they seem to have the biggest impact on higher alcohol and related metabolite production of the 10 selected genes and are likely to play a direct role in the Ehrlich pathway. The distant position of $\triangle p r o 2$ might be explained by the fact it is the only reductase included in the set of selected genes and is therefore completely different from the rest of the group. This is also reflected in the actual GC data where effects dissimilar to the rest of the genes are observed. It is thus likely that $\Delta p r o 2$ could play a direct part in the metabolism of the higher alcohols and volatile branched chain fatty acids, but in a completely unrelated fashion as the other selected genes.

\section{Discussion}

From the evidence presented here, it is clear that deletion of the selected genes has a significant impact on the catabolism of branched chain amino acids via the Ehrlich pathway into higher alcohols and volatile acids, as well as 

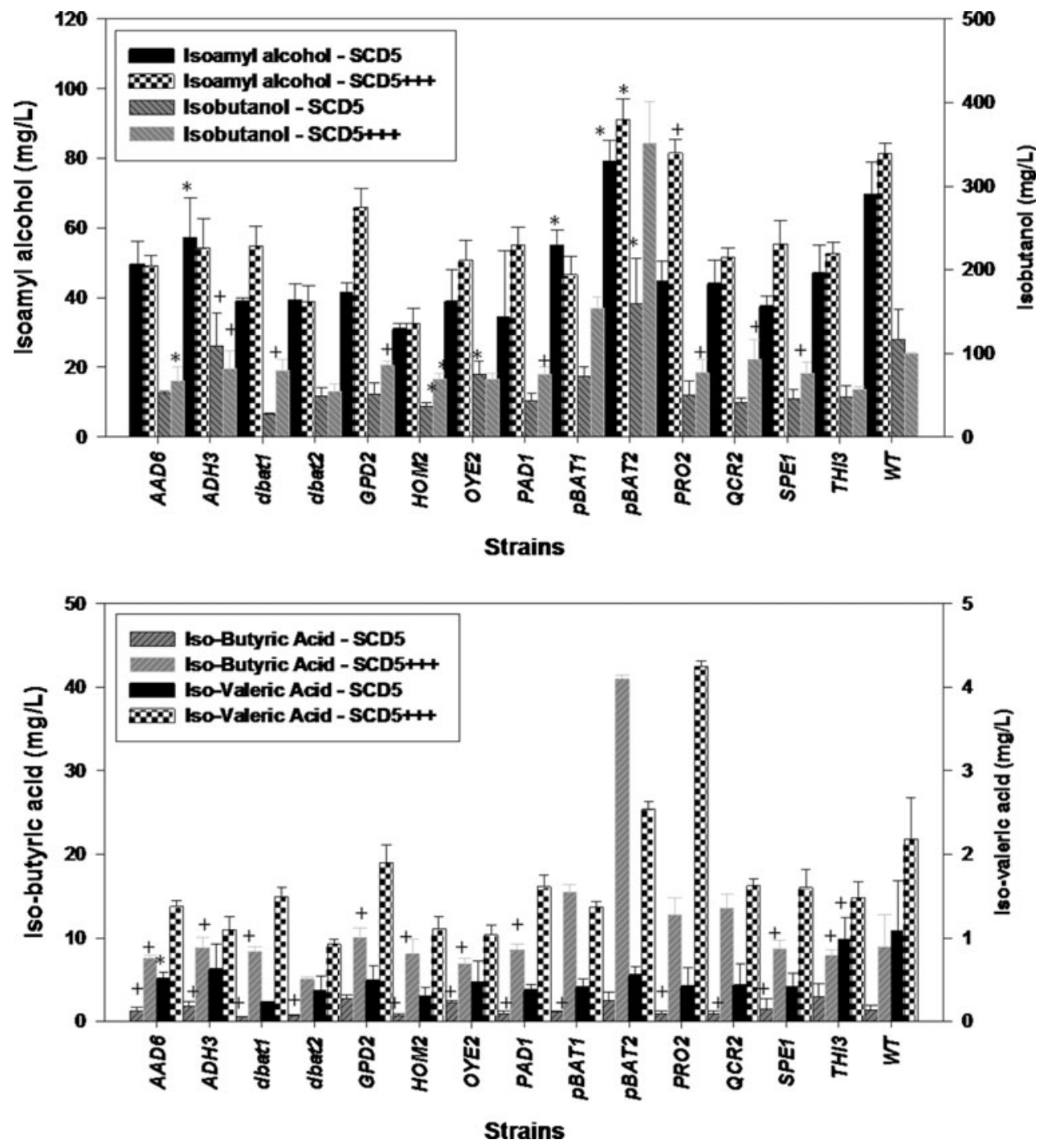

Fig. 6 GC FID analysis of the concentrations of the two major higher alcohols formed from the branched chain amino acids, isoamyl alcohol and isobutanol, as well as two major higher volatile fatty acids, iso butyric and iso valeric acid, formed after cells were grown for 1 week in SCD5 or SCD5+++ medium. The graph shows the differences between the deletion strains, certain control strains, and the wild type. In the top panel, black bars indicate concentrations of isoamyl alcohol formed from cells grown in SCD5 medium and chequered bars the concentration of isoamyl alcohol from SCD5+++ grown strains. The dark and light gray bars indicate the same differences in growth medium for levels of isobutanol produced. In the

the general aroma profile of the yeast strain. However, it is also clear that not all genes are directly involved in these chemical reactions and some exert their effect via indirect means, with the maintenance of redox homeostasis being the most obvious role player. Indeed, two of the genes are involved in major redox maintenance by regenerating $\mathrm{NAD}^{+}$during fermentative conditions. GPD2 encodes for the NAD-dependent glycerol 3-phosphate dehydrogenase located in the cytosol and mitochondrion (Albertyn et al. bottom panel, black bars indicate concentrations of iso butyric acid formed from cells grown in SCD5 medium and chequered bars the concentration of iso butyric acid from SCD5+++ grown strains. The dark and light gray bars indicate the same differences in growth medium for levels of iso valeric acid produced. The experiments were performed in triplicate and the error bars are shown. The asterisk on top of the bars $(*)$ denote cases of statistical significance $(p<0.001)$ from the wild type after one way ANOVA and a Holm Sidak post test, while unmarked bars indicate statistical significance with a $p$ value of less than 0.05 , but more than 0.001 . Crosses $(+)$ denote cases of no statistical difference

1992). Gpd2p catalyzes the conversion of dihydroxy acetone phosphate to glycerol-3-phosphate with the simultaneous oxidation of NADH to $\mathrm{NAD}^{+}$(Cronwright et al. 2002; Larsson et al. 1998; Pahlman et al. 2001, 2002). $A D H 3$ encodes for the mitochondrial alcohol dehydrogenase isozyme III (Young and Pilgrim 1985). Adh3p is involved in the NADH-dependent reduction of acetaldehyde to ethanol during glucose fermentation and plays a part in the ethanol-acetaldehyde redox shuttle that helps in 
a

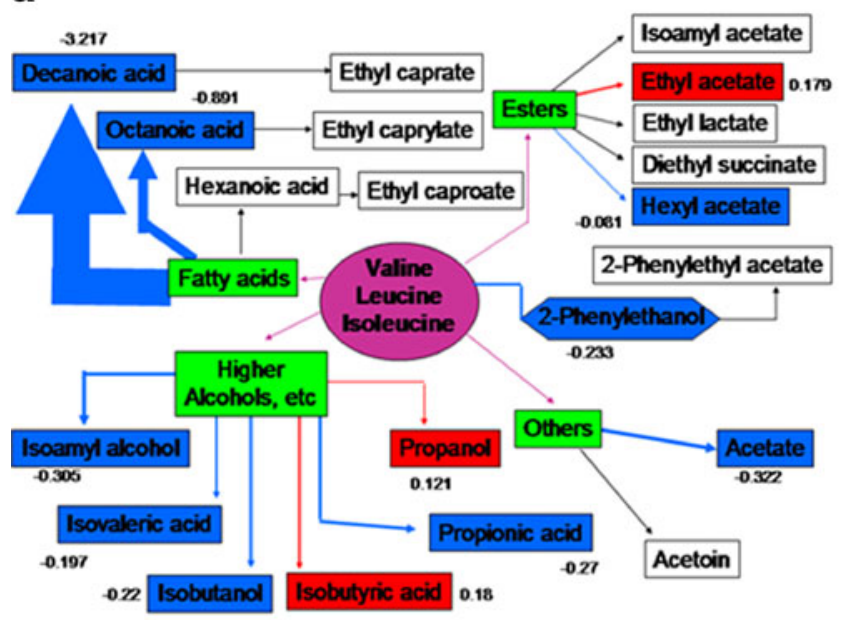

C

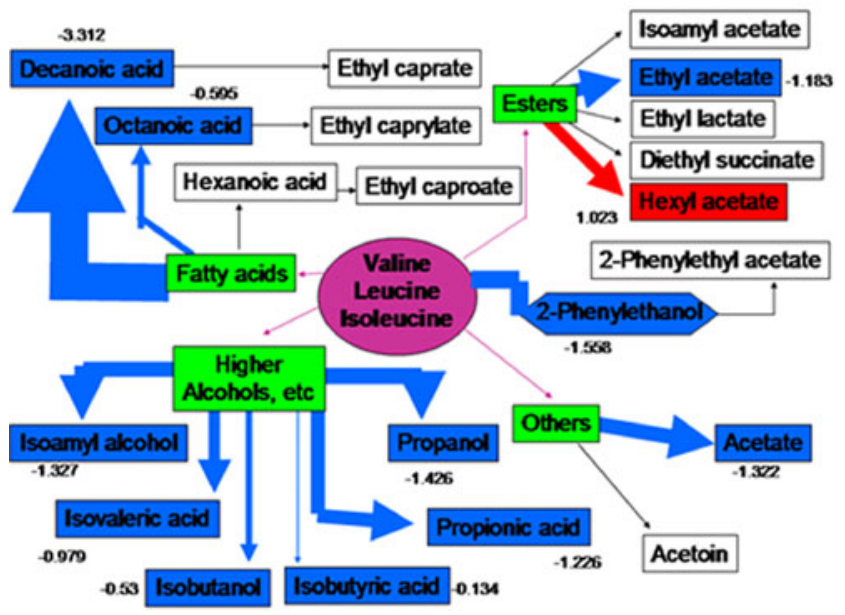

Fig. 7 Schematic representation of the effect of certain selected gene deletions on the yeast aroma profile in cells grown in SCD5+++ medium. Red indicates an increase and blue a decrease, while the

maintaining the redox balance of the mitochondrion (Bakker et al. 2000, 2001). Previous authors (Dickinson et al. 2003) investigated whether $A D H 3$ can play a role in the formation of higher alcohols and indeed found that any of the ADH genes can fulfill this function. Interestingly, in this study, no significant effect was seen on higher alcohol production when any of the other ADH genes were deleted. The results clearly demonstrate that the Ehrlich pathway is highly sensitive to the general redox balance of the cell and that the redox duality of the pathway can be useful in overcoming perturbations caused by the deletion of important redox-regulating genes. In addition, the data suggest that the other $A D H$ genes do not normally play a direct role within this pathway.

Three other dehydrogenases were also among the selected genes investigated. OYE2 is a well-conserved NADPH dehydrogenase, but its exact function is still unclear b

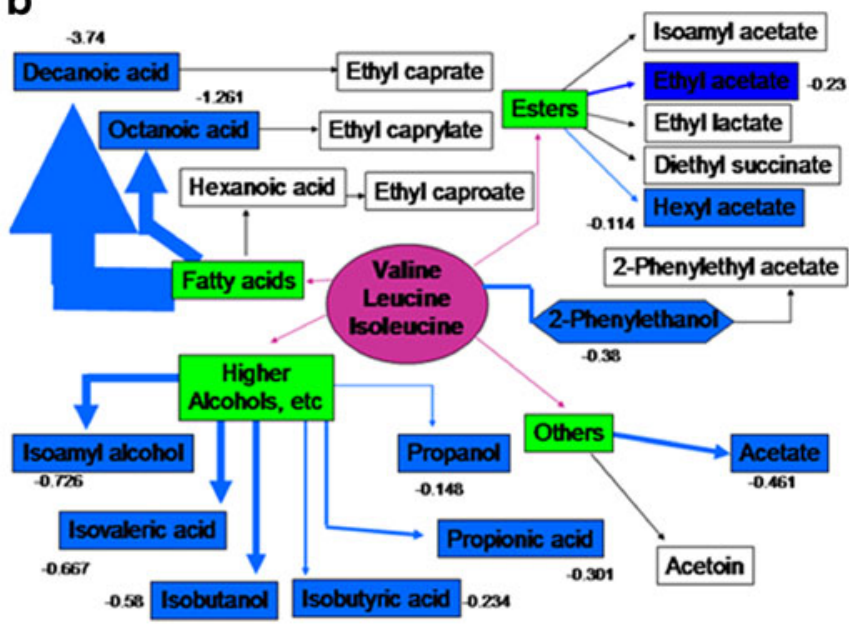

d

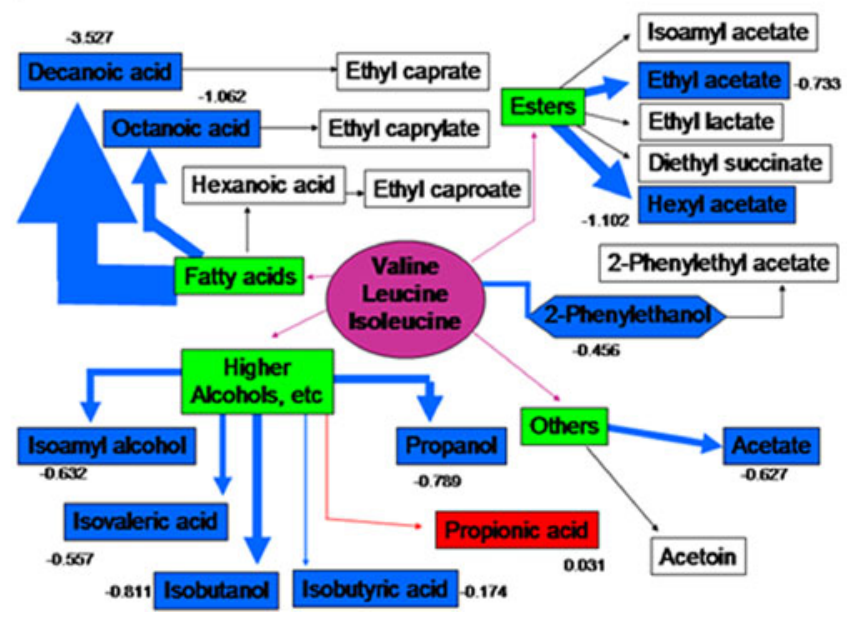

arrow thickness gives an indication of the magnitude of the effect in $\log 2$. The panels correspond to the following deletion strains: a $\Delta g p d 2, \mathbf{b} \Delta a a d 6, \mathbf{c} \Delta h o m 2, \mathbf{d} \Delta t h i 3$

(Matthews and Massey 1968; Saito et al. 1991; Stott et al. 1993; Niino et al. 1995). It is able to oxidize both NADPH and NADH, although less efficiently, and various phenolic compounds may be able to bind to it (Brown et al. 1998, 2002). Other researchers have found that Oye $2 p$ can associate with actin and protect the actin cytoskeleton from oxidative stress (Haarer and Amberg 2004). However, a study that impacts on the results from this study found that Oye $2 p$ can reduce acrolein and other $\alpha, \beta$-unsaturated aldehydes (Trotter et al. 2006). This might directly implicate OYE2 in the Ehrlich pathway. Indeed, deletion of OYE2 leads to notable decreases in the levels of isoamyl alcohol, isobutanol, and iso-valeric acid, but large increases in iso-butyric acid concentrations were sometimes observed. It would seem that the cell has to maintain a fine balance between redox maintenance and the removal of the potentially toxic aldehydes formed during the Ehrlich reaction. 
A
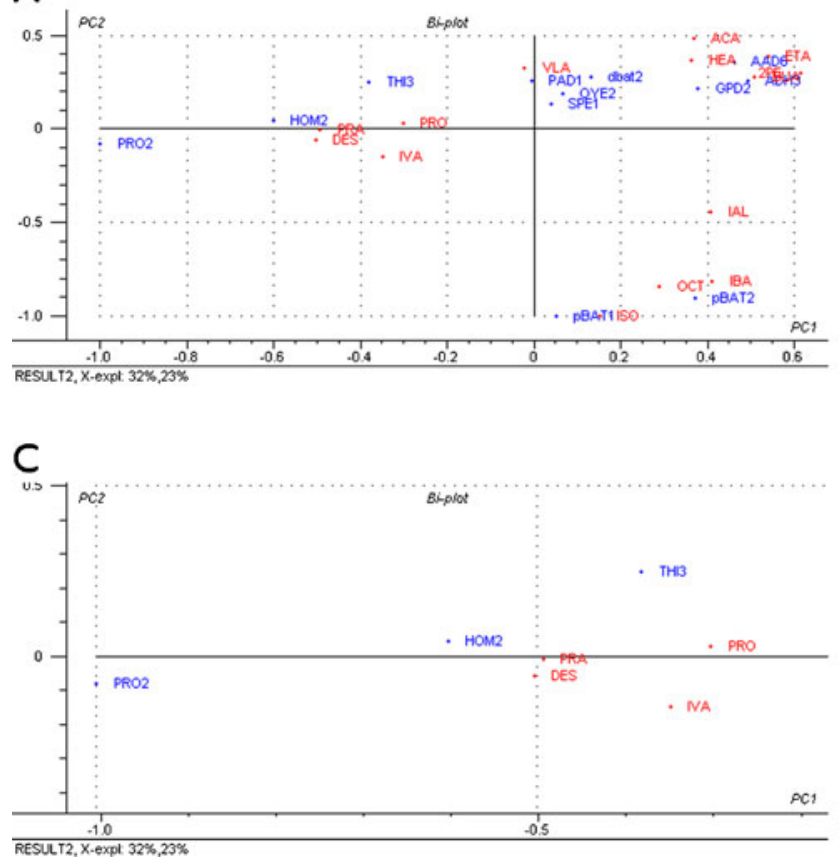

Fig. 8 Bi plot of component 1 vs. component 2 of a principal component analysis of the gas chromatographic dataset of the 10 candidate genes as well as various control genes. a The bi plot in totality, while $\mathbf{b}$ focuses on the right side of PC1 and $\mathbf{c}$ shows an enhanced view of the left side of PC1. d The bottom right side of the bi plot. The abbreviations in the figures represent aroma related

The data suggest that the other two dehydrogenase genes may be directly involved in the Ehrlich pathway. AAD6 is a putative aryl-alcohol dehydrogenase with high sequence similarity to an enzyme in the lignin-degrading fungus Phanerochaete chrysosporium (Delneri et al. 1999a, b). During the breakdown of lignin by this white rot fungus, various peroxidases are very active, leading to products with many aldehyde, quinine, and acidic groups which must be reduced by the $A A D$ gene products (Reiser et al. 1994). However, while Saccharomyces cerevisiae contains seven $A A D$ genes (Delneri et al. 1999b), the aryl-alcohol dehydrogenase activity found in this yeast appears to be independent of these genes and their substrate is still unknown (Delneri et al. 1999a). Dickinson et al. (2003) speculated that these genes may be involved in the Ehrlich pathway, but studies with a strain deleted in all seven $A A D$ genes showed no effect on the level of isoamyl alcohol produced (Dickinson et al. 2003). This is in contrast to the present study which shows decreases in the level of isoamyl alcohol, isobutanol, and iso-valeric acid for the single aad6 mutant. These contradictory findings may be explained by the composition of the medium: Dickinson et al. (2003) used a minimal medium with only leucine, while our media contained all amino acids. Furthermore, our data strongly suggest that of the seven $A A D$ genes, $A A D 6$ is the main enzyme involved in the Ehrlich pathway
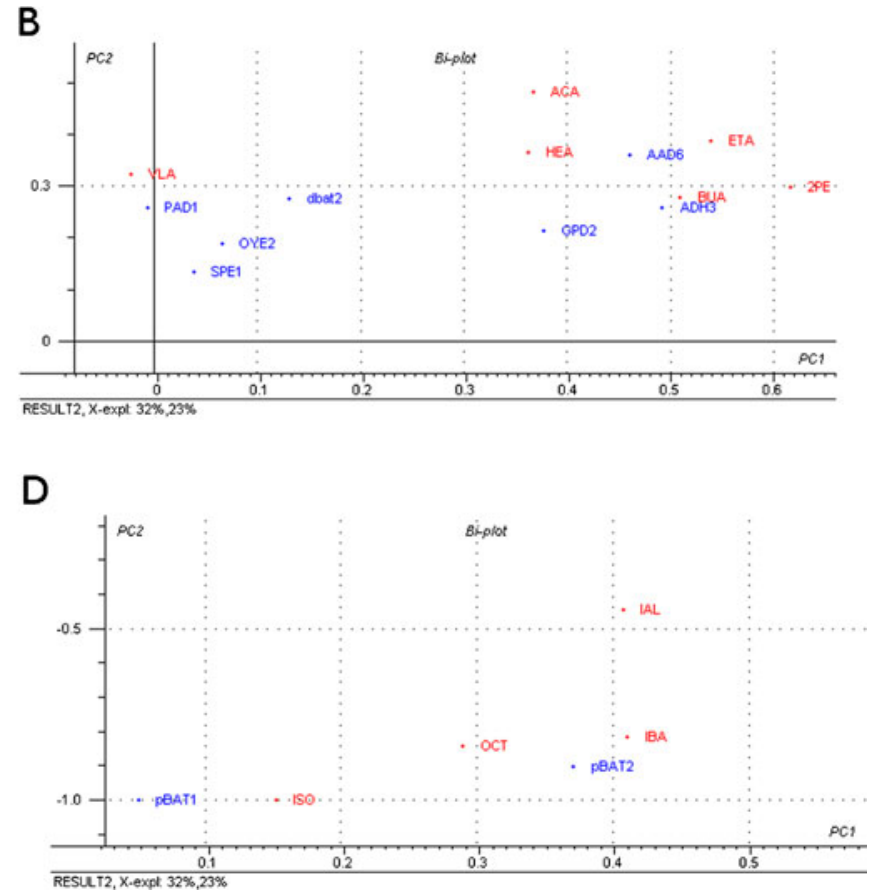

metabolites as follows: $2 P E 2$ phenyl ethanol, $A C A$ acetic acid, $B U A$ butyric acid, DES diethyl succinate, ETA ethyl acetate, $H E A$ hexanoic acid, IAL isoamyl alcohol, ISO isobutanol, IBA iso butyric acid, IVA iso valeric acid, $O C T$ octanoic acid, $P R O$ propanol, $P R A$ propionic acid, $V L A$ valeric acid

Deletion of the HOM2 gene leads to the greatest reduction in the concentration of higher alcohols and volatile acids, suggesting that $H O M 2$ plays a central and direct role in the formation of both the higher alcohols and volatile acids. HOM 2 encodes an aspartic $\beta$ semi-aldehyde dehydrogenase, which catalyzes the second step in the pathway for methionine and threonine biosynthesis. This step involves the reduction of aspartyl-4-phosphate to aspartate-4-semi-aldehyde, using NADPH (Bareich et al. 2003; Arevalo-Rodriguez et al. 2004). However, when de Robichond-Szulmajster et al. (1965) measured the enzymatic activities of this enzyme, they did so in the reverse direction (de Robichond-Szulmajster et al. 1965), giving credence to the direct role that this enzyme plays in the final reaction of the Ehrlich pathway and the ability of this enzyme to reduce or oxidize the aldehyde precursor.

$P R O 2$ deletion led to some interesting results regarding the production of these compounds when compared to the other selected strains. The levels of the volatile fatty acids are affected positively when this gene is deleted in strains grown on medium supplemented with branched chain amino acids (SCD5+++), suggesting that Pro2p plays a different role in the Ehrlich pathway than the other selected genes. This is also reflected in the fact that PRO2 encodes for a gamma-glutamyl phosphate reductase and catalyzes 
the second step in proline biosynthesis (Brandriss 1979). More specifically, it is responsible for the conversion of $\gamma$ glutamyl phosphate and NADPH into L-glutamate- $\gamma$-semialdehyde and NADP ${ }^{+}$(Tomenchok and Brandriss 1987).

The last three selected genes are all classified as decarboxylases and could catalyze the conversion of the $\alpha$-keto acid into its corresponding aldehyde in the Ehrlich reaction. PADI and SPE1 are both thought to encode decarboxylases that can act promiscuously and accept various chemical compounds as substrates. The gene product of $P A D 1$ is a phenylacrylic acid decarboxylase and confers resistance to cinnamic acid and other phenylacrylic acids (Larsson et al. 2001). Pad1p has also been shown to decarboxylate phenolic or aromatic carboxylic acids to their corresponding vinyl derivatives (Smit et al. 2003). SPE1 encodes an ornithine decarboxylase which catalyzes the first step in polyamine biosynthesis in the cytoplasm, more specifically the decarboxylation of ornithine into putrescine (Balasundaram et al. 1994; Fonzi and Sypherd 1987; McNemar et al. 1997). It is degraded in a proteasome-dependent manner in the presence of excess polyamines (Gandre and Kahana 2002; Hoyt et al. 2003; Toth and Coffino 1999). Other authors have recently shown that the enzymes that decarboxylate aromatic amino acids, i.e., $A R O 10$, can play a role in the Ehrlich pathway and may also decarboxylate non-aromatic amino acids (Vuralhan et al. 2005). This might indicate that Padlp and Spelp display similar promiscuity towards their substrate and can directly decarboxylate the $\alpha$-keto acids formed after transamination of the branched chain amino acids.

The last gene identified in the screening procedure has previously been suggested to be involved with the Ehrlich pathway. THI3 (also known as KIDI) encodes a probable decarboxylase which plays a role as a regulatory protein of the enzymes involved in thiamine biosynthesis (Hohmann and Meacock 1998; Mojzita and Hohmann 2006; Nishimura et al. 1992). Thi3p may also play a role in the catabolism of amino acids to long-chain and complex alcohols (Dickinson 2000; Dickinson et al. 2003). This last scenario fits well with the data from this study and the results suggest that THI3 indeed plays a direct role in the Ehrlich pathway.

In summary, the data suggest that several of the selected genes play a direct role within the aroma production network of $S$. cerevisiae. Interestingly, our screen identified few of the genes that had previously been suggested to play a role in aroma production (Ter Schure et al. 1998; Yoshimoto et al. 2001; Dickinson et al. 2003; Vuralhan et al. 2003, 2005). Previous suggestions in this regard, however, were based on the directed analysis of specific genes, frequently analyzed for different aspects of their phenotype. As far as we are aware, this is the first description of a large-scale screening approach focusing directly on aroma production as a selection criteria. Several of the genes identified here are therefore highly likely to be directly involved in these pathways and should be excellent targets for biotechnological improvement of aroma production capacity of industrial strains of $S$. cerevisiae. However, further studies are needed to clarify their specific roles.

\section{References}

Albertyn J, van Tonder A, Prior BA (1992) Purification and character ization of glycerol 3 phosphate dehydrogenase of Saccharomyces cerevisiae. FEBS Lett 308(2):130 132

Ardö Y (2006) Flavour formation by amino acid catabolism. Biotechnol Adv 24:238 242

Arevalo Rodriguez M, Pan X, Boeke JD, Heitman J (2004) FKBP12 controls aspartate pathway flux in Saccharomyces cerevisiae to prevent toxic intermediate accumulation. Eukaryot Cell 3 (5):1287 1296

Ausubel F, Brent R, Kingston R, Moore D, Seidman J, Smith J, Struhl $\mathrm{K}$ (eds) (2003) Current protocols in molecular biology. Wiley, New York

Bakker BM, Bro C, Kotter P, Luttik MA, van Dijken JP, Pronk JT (2000) The mitochondrial alcohol dehydrogenase Adh3p is involved in a redox shuttle in Saccharomyces cerevisiae. J Bacteriol 182(17):4730 4737

Bakker BM, Overkamp KM, van Maris AJ, Kotter P, Luttik MA, van Dijken JP, Pronk JT (2001) Stoichiometry and compartmentation of NADH metabolism in Saccharomyces cerevisiae. FEMS Microbiol Rev 25(1):15 37

Balasundaram D, Xie QW, Tabor CW, Tabor H (1994) The presence of an active $\mathrm{S}$ adenosylmethionine decarboxylase gene increases the growth defect observed in Saccharomyces cerevisiae mutants unable to synthesize putrescine, spermidine, and spermine. J Bacteriol 176(20):6407 6409

Bareich DC, Nazi I, Wright GD (2003) Simultaneous in vitro assay of the first four enzymes in the fungal aspartate pathway identifies a new class of aspartate kinase inhibitor. Chem Biol 10(10):967 973

Bauer FF, Pretorius IS (2000) Yeast stress response and fermentation efficiency: how to survive the making of wine. S Afr J Enol Vitic 21:27 51

Beltran G, Novo M, Rozes N, Mas A, Guillamon JM (2004) Nitrogen catabolite repression in Saccharomyces cerevisiae during wine fermentations. FEMS Yeast Res 4(6):625 632

Ben Yosef T, Eden A, Benvenisty N (1998) Characterization of murine BCAT genes: Bcat1, a c Myc target, and its homolog, Bcat2. Mamm Genome 9(7):595 597

Boulton R, Singleton V, Bisson L, Kunkee R (1995) Principles and practices of winemaking. Chapman Hall, New York

Brandriss M (1979) Isolation and preliminary characterization of Saccharomyces cerevisiae proline auxotrophs. J Bacteriol 138 (3):816 822

Brown BJ, Deng Z, Karplus PA, Massey V (1998) On the active site of old yellow enzyme. Role of histidine 191 and asparagine 194. J Biol Chem 273(49):32753 32762

Brown BJ, Hyun JW, Duvvuri S, Karplus PA, Massey V (2002) The role of glutamine 114 in old yellow enzyme. J Biol Chem 277 (3):2138 2145

Chen EC H (1977) The relative contribution of Ehrlich and biosynthetic pathways to the formation of fusel alcohols. J Am Soc Brew Chem 36(1):39 43

Cronwright GR, Rohwer JM, Prior BA (2002) Metabolic control analysis of glycerol synthesis in Saccharomyces cerevisiae. Appl Environ Microbiol 68(9):4448 4456 
Dahlquist KD, Salomonis N, Vranizan K, Lawlor SC, Conklin BR (2002) GenMAPP, a new tool for viewing and analyzing microarray data on biological pathways. Nat Genet 31(1):19 20

de Robichond Szulmajster H, Surdin Y, Mortimer R (1965) Genetic and biochemical studies on genes controlling the synthesis of threonine and methionine in Saccharomyces. Genetics 53:609 619

Delneri D, Gardner DC, Bruschi CV, Oliver SG (1999a) Disruption of seven hypothetical aryl alcohol dehydrogenase genes from Saccharomyces cerevisiae and construction of a multiple knock out strain. Yeast 15(15):1681 1689

Delneri D, Gardner DC, Oliver SG (1999b) Analysis of the seven member AAD gene set demonstrates that genetic redundancy in yeast may be more apparent than real. Genetics 153(4):1591 1600

Dickinson JR (2000) Pathways of leucine and valine catabolism in yeast. Methods Enzymol 324:80 92

Dickinson JR, Norte V (1993) A study of branched chain amino acid aminotransferase and isolation of mutations affecting the catab olism of branched chain amino acids in Saccharomyces cerevi siae. FEBS Lett 326(1,2,3):29 32

Dickinson JR, Lanterman M, Danner D, Pearson B, Sanz P, Harrison SJ, Hewlins MJ (1997) A ${ }^{13} \mathrm{C}$ nuclear magnetic resonance investigation of the metabolism of leucine to isoamyl alcohol in Saccharomyces cerevisiae. J Biol Chem 272(43):26871 26878

Dickinson JR, Harrison SJ, Hewlins MJ (1998) An investigation of the metabolism of valine to isobutyl alcohol in Saccharomyces cerevisiae. J Biol Chem 273(40):25751 25756

Dickinson JR, Harrison SJ, Dickinson JA, Hewlins MJ (2000) An investigation of the metabolism of isoleucine to active amyl alcohol in Saccharomyces cerevisiae. J Biol Chem 275 (15):10937 10942

Dickinson JR, Salgado L, Hewlins MJ (2003) The catabolism of amino acids to long chain and complex alcohols in Saccharomyces cerevisiae. J Biol Chem 278(10):8028 8034

Doniger SW, Salomonis N, Dahlquist KD, Vranizan K, Lawlor SC, Conklin BR (2003) MAPPFinder: using Gene Ontology and GenMAPP to create a global gene expression profile from microarray data. Genome Biol 4(1):R7

Eden A, Simchen G, Benvenisty N (1996) Two yeast homologs of ECA39, a target for c Myc regulation, code for cytosolic and mitochondrial branched chain amino acid aminotransferases. J Biol Chem 271(34):20242 20245

Eden A, Van Nedervelde L, Drukker M, Benvenisty N, Debourg A (2001) Involvement of branched chain amino acid aminotrans ferases in the production of fusel alcohols during fermentation in yeast. Appl Microbiol Biotechnol 55(3):296 300

Estevez P, Gil M, Falque E (2004) Effects of seven yeast strains on the volatile composition of Palomino wines. Int J Food Sci Technol 39:61 69

Fonzi WA, Sypherd PS (1987) The gene and the primary structure of ornithine decarboxylase from Saccharomyces cerevisiae. J Biol Chem 262(21):10127 10133

Gandre S, Kahana C (2002) Degradation of ornithine decarboxylase in Saccharomyces cerevisiae is ubiquitin independent. Biochem Biophys Res Commun 293(1):139 144

Grauslund M, Didion T, Kielland Brandt MC, Andersen HA (1995) BAP2, a gene encoding a permease for branched chain amino acids in Saccharomyces cerevisiae. Biochim Biophys Acta 1269 (3):275 280

Haarer BK, Amberg DC (2004) Old yellow enzyme protects the actin cytoskeleton from oxidative stress. Mol Biol Cell 15(10):4522 4531

Hazelwood L, Daran JM, Van Maris AJ, Pronk JT, Dickinson JA (2008) The Ehrlich pathway for fusel alcohol production: a century of research on Saccharomyces cerevisiae metabolism. Appl Environ Microbiol 74:2259 2266
Hohmann S, Meacock PA (1998) Thiamin metabolism and thiamin diphosphate dependent enzymes in the yeast Saccharomyces cer evisiae: genetic regulation. Biochim Biophys Acta 1385(2):201 219

Hoyt MA, Zhang M, Coffino P (2003) Ubiquitin independent mechanisms of mouse ornithine decarboxylase degradation are conserved between mammalian and fungal cells. J Biol Chem 278(14):12135 12143

Jansen M, Veurink JH, Euverink GJ, Dijkhuizen L (2003) Growth of the salt tolerant yeast Zygosaccharomyces rouxii in microtiter plates: effects of $\mathrm{NaCl}, \mathrm{pH}$ and temperature on growth and fusel alcohol production from branched chain amino acids. FEMS Yeast Res 3(3):313 318

Kispal G, Steiner H, Court DA, Rolinski B, Lill R (1996) Mitochondrial and cytosolic branched chain amino acid trans aminases from yeast, homologs of the myc oncogene regulated Eca39 protein. J Biol Chem 271(40):24458 24464

Lambrechts MG, Pretorius IS (2000) Yeast and its importance to wine aroma. S Afr J Enol Vitic 21:97 129

Larsson C, Pahlman IL, Ansell R, Rigoulet M, Adler L, Gustafsson L (1998) The importance of the glycerol 3 phosphate shuttle during aerobic growth of Saccharomyces cerevisiae. Yeast 14(4):347 357

Larsson S, Nilvebrant NO, Jonsson LJ (2001) Effect of overexpression of Saccharomyces cerevisiae Pad1p on the resistance to phenylacrylic acids and lignocellulose hydrolysates under aerobic and oxygen limited conditions. Appl Microbiol Biotechnol 57(1 2):167 174

Lilly M, Lambrechts MG, Pretorius IS (2000) Effect of increased yeast alcohol acetyltransferase activity on flavor profiles of wine and distillates. Appl Environ Microbiol 66(2):744 753

Lilly M, Bauer FF, Styger G, Lambrechts MG, Pretorius IS (2006) The effect of increased branched chain amino acid transaminase activity in yeast on the production of higher alcohols and on the flavour profiles of wine and distillates. FEMS Yeast Res 6(5):726 743

Majdak A, Herjavec S, Orlic S, Redzepovic S, Mirosevic N (2002) Comparison of wine aroma compounds produced by Saccharomyces paradoxus and Saccharomyces cerevisiae strains. Food Technol Biotechnol 40(2):103 109

Mateo J, Jimenez M, Pastor A, Huerta T (1998) Influence of the inoculation time of high sugar content must on the formation of wine aroma. World J Microbiol Biotechnol 14:357 363

Matthews R, Massey V (1968) Isolation of old yellow enzyme in free and complexed forms. J Biol Chem 244(7):1779 1786

McNemar MD, Gorman JA, Buckley HR (1997) Isolation and sequence of the gene encoding ornithine decarboxylase, SPE1, from Candida albicans by complementation of a spe1 delta strain of Saccharomyces cerevisiae. Yeast 13(14):1383 1389

Mojzita D, Hohmann S (2006) Pdc2 coordinates expression of the THI regulon in the yeast Saccharomyces cerevisiae. Mol Genet Genomics 276(2):147 161

Niino YS, Chakraborty S, Brown BJ, Massey V (1995) A new old yellow enzyme of Saccharomyces cerevisiae. J Biol Chem 270 (5):1983 1991

Nishimura H, Kawasaki Y, Kaneko Y, Nosaka K, Iwashima A (1992) A positive regulatory gene, THI3, is required for thiamine metabolism in Saccharomyces cerevisiae. J Bacteriol 174 (14):4701 4706

Pahlman IL, Gustafsson L, Rigoulet M, Larsson C (2001) Cytosolic redox metabolism in aerobic chemostat cultures of Saccharomy ces cerevisiae. Yeast 18(7):611 620

Pahlman IL, Larsson C, Averet N, Bunoust O, Boubekeur S, Gustafsson L, Rigoulet M (2002) Kinetic regulation of the mitochondrial glycerol 3 phosphate dehydrogenase by the exter nal NADH dehydrogenase in Saccharomyces cerevisiae. J Biol Chem 277(31):27991 27995

Prohl C, Kispal G, Lill R (2000) Branched chain amino acid trans aminases of yeast Saccharomyces cerevisiae. Methods Enzymol $324: 365375$ 
Quain D (1988) Studies on yeast physiology impact of fermentation performance and product quality. J Inst Brew 94:315 323

Reiser J, Muheim A, Hardegger M, Frank G, Fiechter A (1994) Aryl alcohol dehydrogenase from the white rot fungus Phanerochaete chrysosporium. Gene cloning, sequence analysis, expression, and purification of the recombinant enzyme. J Biol Chem 269 (45):28152 28159

Romano P, Fiore C, Paraggio M, Caruso M, Capece A (2003) Function of yeast species and strains in wine flavour. Int J Food Microbiol 86(1 2):169 180

Rossouw D, Naes T, Bauer FF (2008) Linking gene regulation and the exo metabolome: a comparative transcriptomics approach to identify genes that impact on the production of volatile aroma compounds in yeast. BMC Genomics 9:530

Saito K, Thiele DJ, Davio M, Lockridge O, Massey V (1991) The cloning and expression of a gene encoding Old Yellow Enzyme from Saccharomyces carlsbergensis. J Biol Chem 266 (31):20720 20724

Sambrook J, Fritsch E, Maniatis T (1989) Molecular cloning: a laboratory manual, 2nd edn. Cold Spring Harbor Laboratory Press, Cold Spring Harbor

Smit A, Cordero Otero RR, Lambrechts MG, Pretorius IS, Van Rensburg P (2003) Enhancing volatile phenol concentrations in wine by expressing various phenolic acid decarboxylase genes in Saccharomyces cerevisiae. J Agric Food Chem 51(17):4909 4915

Stott K, Saito K, Thiele DJ, Massey V (1993) Old Yellow Enzyme. The discovery of multiple isozymes and a family of related proteins. J Biol Chem 268(9):6097 6106

Ter Schure EG, Flikweert MT, Van Dijken JP, Pronk JT, Verrips CT (1998) Pyruvate decarboxylase catalyzes decarboxylation of branched chain 2 oxo acids but is not essential for fusel alcohol production by Saccharomyces cerevisiae. Appl Environ Micro biol 64(4):1303 1307

Thierry A, Maillard M B (2002) Production of cheese flavour compounds derived from amino acid catabolism by Propioni bacterium freudenreichii. Lait 82:17 32

Thierry A, Maillard M B, Yvon M (2002) Conversion of L leucine to isovaleric acid by Propionibacterium freudenreichii TL 34 and ITGP23. Appl Environ Microbiol 68(2):608 615
Tomenchok D, Brandriss M (1987) Gene enzyme relationships in the proline biosynthesis pathway of Saccharomyces cerevisiae. J Bacteriol 169(12):5364 5372

Toth C, Coffino P (1999) Regulated degradation of yeast ornithine decarboxylase. J Biol Chem 274(36):25921 25926

Trelea I, Titica M, Corrieu G (2004) Dynamic optimisation of the aroma production in brewing fermentation. J Process Control 14:1 14

Trotter EW, Collinson EJ, Dawes IW, Grant CM (2006) Old yellow enzymes protect against acrolein toxicity in the yeast Saccharomyces cerevisiae. Appl Environ Microbiol 72(7):4885 4892

Van Dijken JP, Scheffers W (1986) Redox balances in the metabolism of sugars by yeasts. FEMS Microbiol Rev 32:199 224

Vanderhaegen B, Neven H, Coghe S, Verstrepen K, Verachtert H, Derdelinckx G (2003) Evolution of chemical and sensory properties during aging of top fermented beer. J Agric Food Chem 51:6782 6790

Vidrih R, Hribar J (1999) Synthesis of higher alcohols during cider processing. Food Chem 67:287 294

Volbrecht D, Radler F (1973) Formation of higher alcohols by amino acid deficient mutants of Saccharomyces cerevisiae. I. The decomposition of amino acids to higher alcohols. Arch Mikrobiol 94:351 358

Vuralhan Z, Morais MA, Tai SL, Piper MD, Pronk JT (2003) Identification and characterization of phenylpyruvate decarbox ylase genes in Saccharomyces cerevisiae. Appl Environ Micro biol 69(8):4534 4541

Vuralhan Z, Luttik MA, Tai SL, Boer VM, Morais MA, Schipper D, Almering MJ, Kotter P, Dickinson JR, Daran JM, Pronk JT (2005) Physiological characterization of the ARO10 dependent, broad substrate specificity 2 oxo acid decarboxylase activity of Saccha romyces cerevisiae. Appl Environ Microbiol 71(6):3276 3284

Yoshimoto H, Fukushige T, Yonezawa T, Sakai Y, Okawa K, Iwamatsu A, Sone H, Tamai Y (2001) Pyruvate decarboxylase encoded by the PDC1 gene contributes, at least partially, to the decarboxylation of alpha ketoisocaproate for isoamyl alcohol formation in Saccha romyces cerevisiae. J Biosci Bioeng 92(1):83 85

Young ET, Pilgrim D (1985) Isolation and DNA sequence of ADH3, a nuclear gene encoding the mitochondrial isozyme of alcohol dehydrogenase in Saccharomyces cerevisiae. Mol Cell Biol 5 (11):3024 3034 